\title{
Active noise attenuation in ventilation windows
}

\author{
Huahua Huang ${ }^{\text {a) }}$ and Xiaojun Qiu \\ Key Laboratory of Modern Acoustics and Institute of Acoustics, Nanjing University, Nanjing, 210093, \\ People's Republic of China \\ Jian Kang \\ School of Architecture, University of Sheffield, Western Bank, Sheffield S10 2TN, United Kingdom
}

(Received 24 August 2010; revised 4 May 2010; accepted 4 May 2011)

\begin{abstract}
The feasibility of applying active noise control techniques to attenuate low frequency noise transmission through a natural ventilation window into a room is investigated analytically and experimentally. The window system is constructed by staggering the opening sashes of a spaced double glazing window to allow ventilation and natural light. An analytical model based on the modal expansion method is developed to calculate the low frequency sound field inside the window and the room and to be used in the active noise control simulations. The effectiveness of the proposed analytical model is validated by using the finite element method. The performance of the active control system for a window with different source and receiver configurations are compared, and it is found that the numerical and experimental results are in good agreement and the best result is achieved when the secondary sources are placed in the center at the bottom of the staggered window. The extra attenuation at the observation points in the optimized window system is almost equivalent to the noise reduction at the error sensor and the frequency range of effective control is up to $390 \mathrm{~Hz}$ in the case of a single channel active noise control system.
\end{abstract}

(C) 2011 Acoustical Society of America. [DOI: 10.1121/1.3596457]

PACS number(s): 43.50.Ki, 43.50.Rq, 43.55.Br [KVH]

Pages: $176-188$

\section{INTRODUCTION}

Traffic noise is a major environmental problem that has short-term effects and long-term consequences for health. ${ }^{1}$ External noises can be effectively reduced by closing windows, but this is often impossible in summer times and/or in tropical countries where windows need to be frequently kept open to provide sufficient natural ventilation. In terms of energy saving and environmental protection, windows that have good sound insulation while allowing natural ventilation are of great interest to residents, researchers and construction companies.

There have been various attempts to develop suitable windows that could achieve a moderate degree of noise reduction and, meanwhile, retain sufficient ventilation for providing fresh air. Ford and Kerry ${ }^{2}$ and Lawrence and Burgess ${ }^{3}$ investigated the effect of the opening area of windows on the sound reduction index, where it was found that partially opened double glazing is $10 \mathrm{dBA}$ better than partially opened single glazing and double glazing could be opened up to $100 \mathrm{~mm}$ to reach the noise insulation capacity of closed single glazing. Contana ${ }^{4}$ developed a high sound insulation ventilating window, where the window was sealed and a ventilation element, with a fan filled with absorbing materials, was used to supply air flow. The typical weighted sound reduction index of such a system is about $30 \mathrm{~dB}$. Field and Fricke ${ }^{5}$ used a quarterwave resonator to attenuate noise entering buildings through ventilation openings and an extra attenuation (EA) of 6-7 dB was achieved in certain $1 / 3$ octave bands.

\footnotetext{
a) Author to whom correspondence should be addressed. Electronic mail: hahfhy@yahoo.com.cn
}

Recently, Tang et al. ${ }^{6}$ experimentally investigated the sound insulation performance of a specially designed facade device, and the results showed that additional acoustical protection of 12-13 dBA could be gained compared to the conventional window when exposed to traffic noise. Nishimura et $a l^{7}$ presented a model for soundproofing casement windows which are suitable for tropical countries. The locations of input and output as well as the ratio of the input area to the area of the window facade were optimized to prevent higher-order modes. Kang et al. ${ }^{8,9}$ developed a staggered window system, where the opening sashes of a spaced double glazing window were staggered to create a natural ventilation path and prevent direct sound propagation; furthermore transparent microperforated absorbers (MPA) were used along the ventilation path to attenuate external noise. It was found that although the air flow rate was reduced by $2-4$ times for staggered double glazing compared with that for only internal glazing, it can provide sufficient ventilation for comfort with optimized configurations. When the staggered window system is well configured, external noise can be efficiently reduced from $500 \mathrm{~Hz}$ to $8 \mathrm{kHz}$, with better performance than a typical closed single glazing window. However, the performance of the staggered window system is not satisfactory at low frequencies since MPA is mainly effective at mid and high frequencies unless the airspace is sufficiently large.

Active noise control (ANC) techniques are now recognized as an effective way to reduce low frequency noise. ${ }^{10,11}$ Zhang et al. ${ }^{11}$ proposed a model for examining the coherence between reference and error signals, which is essential for actively controlling traffic noise transmission through an open window into a room. Experiments were carried out to 
verify the proposed model. Unfortunately, the results of the noise reduction in the room were not given. Jakob and Möser ${ }^{12,13}$ carried out systematical experiments to investigate the performance of an actively controlled double-glazed window with several configurations. The EA due to the ANC system is about $7 \mathrm{~dB}$ with the feedforward controller and 3-6 dB with the feedback controller. Ise ${ }^{14}$ arranged 16 independent single channel active controllers at an open window to create a virtual acoustical soft boundary, which reflects the incident acoustic energy and accordingly protect the building interior from polluting by external noise. A noise reduction of more than $10 \mathrm{~dB}$ was achieved for the frequency range of $200-700 \mathrm{~Hz}$ at the error sensors. The disadvantage of such a system is that numerous channels of the ANC system will be needed for a relatively large window, leading to less feasibility for practical applications.

In this paper, ANC techniques are explored to extend the frequency range of noise reduction for the staggered window developed by Kang et al. ${ }^{8,9}$ Based on the modal expansion technique, ${ }^{15,16}$ an analytical model for calculating the low frequency sound transmission through the staggered window system into the room is developed first. The key of the analytical model is to formulate the diffraction problem of the staggered window system as a problem of multiple coupled cavities. The sound fields inside the window system and the room are evaluated by using the proposed model, and the results of the sound fields at low frequencies are validated by the finite element method (FEM). The feasibility of applying ANC on such a window system is numerically studied with a number of configurations, and finally experiments are carried out in a 1:2 scale model to verify the numerical simulation results.

\section{THEORY}

\section{A. Model}

A sketch of the staggered window system installed in a room is shown in Fig. 1(a). The external opening sash toward road traffic and the internal opening sash toward the room are denoted as the source side opening ( $\mathrm{SSO}$ ) and the receiver side opening (RSO), respectively. 8,9 The created ventilation path consists of a SSO, a window interior and a RSO. The effect of MPA is ignored in this paper since the sound pressure level (SPL) difference due to MPA is negligible at low frequencies. The window glass, whose sound reduction index is usually higher than $20 \mathrm{~dB}$ at $100 \mathrm{~Hz}$ after a certain thickness, ${ }^{17}$ is assumed to be rigid for simplicity, along with the walls of the accompanying room. This study is restricted to small perturbations, so the effects of the air flow in the ventilation path, which is typically lower than that in mufflers, are neglected for the sake of convenience. With these assumptions, external noise can only propagate into the room through the ventilation path of the staggered window system.

Although FEM is a well-known method for solving the above-stated acoustic problem, it consumes large amounts of memory and has a long calculation time at mid to high frequencies (e.g., $500 \mathrm{~Hz}$ ), especially when an infinite external space needs to be considered for road traffic. Moreover, the
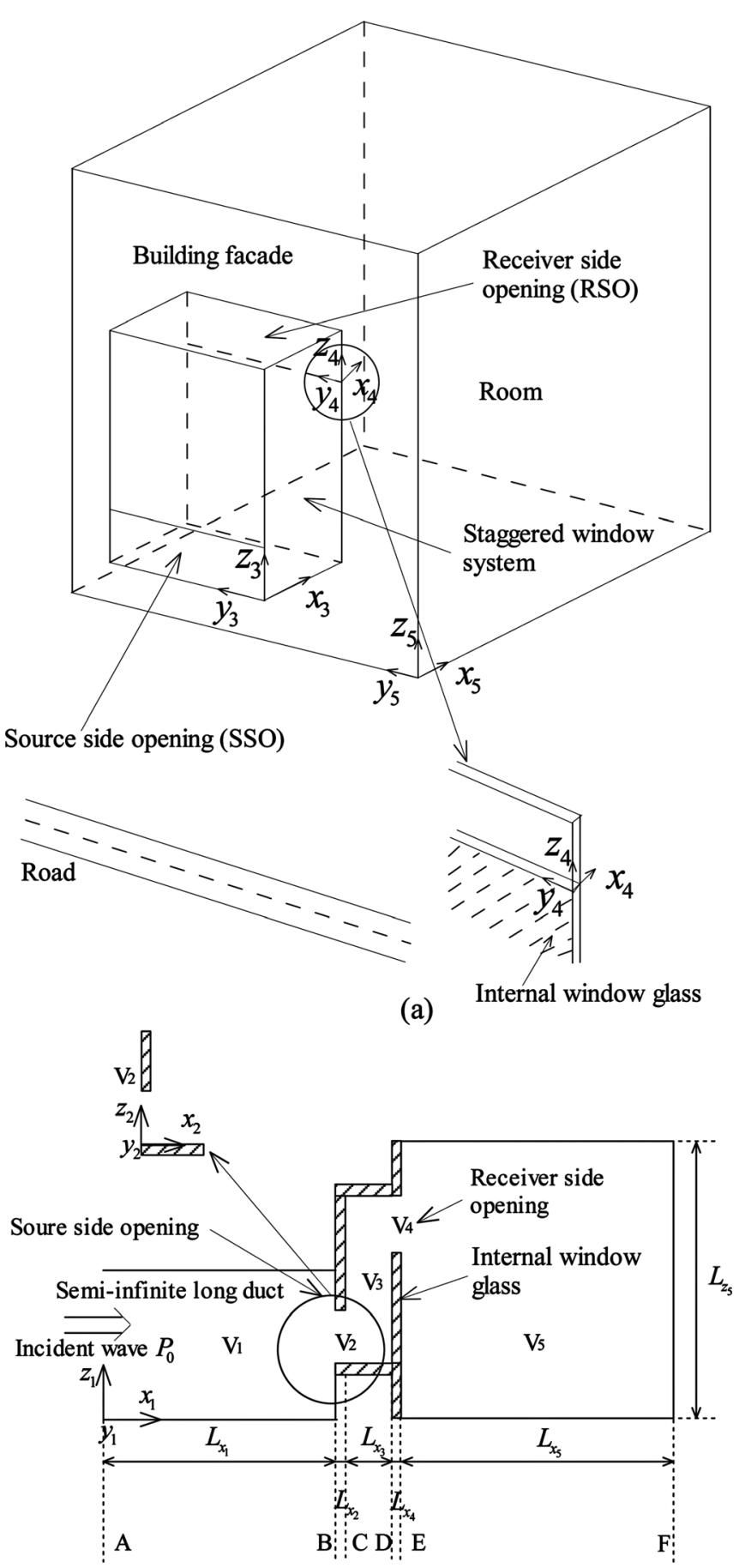

(b)

FIG. 1. Geometry of the staggered window system installed in a room. (a) $3 \mathrm{D}$ perspective. (b) Cross section of the approximate model of (a).

calculation using the FEM is hard to converge for some configurations. Therefore, an analytical model is developed and applied in this research. To solve the problem of sound propagating through the staggered window into the room, the coupled cavities theory based on the modal expansion can be used. ${ }^{15,16}$ The problem of external noise transmission into the staggered window is similar to the problem of calculating the sound inside a balcony with a point source placed at external free space solved by Kropp and Bérillon, ${ }^{18}$ where the opening of the balcony was discretized into rectangular 
elements and they were regarded as pistons which radiate sound into the balcony and external free space, the Rayleigh integral was then carried out over the balcony opening to calculate the sound field in external free space and inside the balcony. A relative simple method was adopted by Nishimura et al., ${ }^{7}$ where the opening toward external free space was treated as a single piston and the effects of external free space were neglected.

As the impact of the staggered window system and the room on external sound field at the road traffic is not the aim in this study, a semi-infinitely long duct is used to simulate external free space, through which the whole system is modeled as a five-cavity ( $\mathrm{V}_{1}$ to $\mathrm{V}_{5}$ ) coupled system, as shown in Fig. 1(b). The method can be viewed as a compromise between the methods used by Kropp and Bérillon ${ }^{18}$ and Nishimura et ll $^{7}$ When the cross section areas of the assumed cavity $\mathrm{V}_{1}$ and the SSO are equivalent, the method here used is almost the same as the one adopted by Nishimura et al. When the cross-sectional area of cavity $\mathrm{V}_{1}$ is infinitely large, it is the case of Kropp and Bérillon. Thus, the analytical model developed here is a more general one, which might be useful for general investigations on sound propagations in the windows configurations studied in this paper. In this paper, the cross section area of the assumed cavity $\mathrm{V}_{1}$ duct is 25 times that of the $\mathrm{SSO}$, which is proved to be sufficiently large for the problem to be solved here. Since road traffic is usually several wavelengths away from windows and the width of windows to be investigated here is generally smaller than one wavelength at low frequency, the incident sound $\left(P_{0}\right)$ is assumed to be planar and with normal incident angle for simplicity.

\section{B. Primary sound field}

To solve the coupled cavities problem where the SSO and the RSO are each regarded as a cavity, the modal expansion method is adopted, ${ }^{15,16}$ which was used in analyzing the transmission loss of a muffler with plenum chambers. ${ }^{16,19,20}$ The cavities of the model are labeled successively as $V_{1}, V_{2}$, $\mathrm{V}_{3}, \mathrm{~V}_{4}$, and $\mathrm{V}_{5}$, corresponding to the assumed semi-infinitely long duct, the SSO, the staggered window, the RSO and the room, respectively, as shown in Fig. 1(b). Five coordinate systems are established with their origins respectively at the right front bottom corner of the five cavities, namely $\left(x_{1}, y_{1}, z_{1}\right)$ and $\left(x_{2}, y_{2}, z_{2}\right)$, as shown in Fig. 1(b), and $\left(x_{3}, y_{3}, z_{3}\right)$, $\left(x_{4}, y_{4}, z_{4}\right)$ and $\left(x_{5}, y_{5}, z_{5}\right)$, as illustrated in Fig. 1(a). Although these five coordinate systems can be represented by only one Cartesian coordinate system, they are still respectively marked for convenient mathematical expression. Five $y-z$ planes, respectively located in the origins of the five coordinate systems are labeled as A, B, C, D, and E. Plane F is at the rear wall of cavity $\mathrm{V}_{5}$, as shown in Fig. 1(b). The dimensions along the $x$ axis, $y$ axis, and $z$ axis are, respectively termed as the length $\left(L_{x}\right)$, width $\left(L_{y}\right)$ and height $\left(L_{z}\right)$ of a cavity within the following context for simplicity.

The unknown sound pressures in the five cavities are expressed by the superposition of modes. For the $l$ th rectangular cavity, with the simple harmonic time-dependence factor $\exp (j \omega t)$ omitted, it can be written as

$$
\begin{aligned}
p_{l}^{P}=p_{l}^{i}+p_{l}^{r}= & \sum_{m_{l}=0}^{\infty} \sum_{n_{l}=0}^{\infty} \varphi_{l}^{m_{l} n_{l}}\left(y_{l}, z_{l}\right)\left[P_{i_{l}}^{m_{l} n_{l}} \exp \left(-j k_{x_{l}}^{m_{l} n_{l}} x_{l}\right)\right. \\
& \left.+P_{r_{l}}^{m_{l} n_{l}} \exp \left(j k_{x_{l}}^{m_{l} n_{l}} x_{l}\right)\right](l=1,2, \ldots, 5),
\end{aligned}
$$

where $p_{l}^{i}$ and $p_{l}^{r}$ are the sounds propagating in the positive and negative $x$ directions inside the $l$ th cavity, respectively. $P_{i_{l}}^{m_{l} n_{l}}$ and $P_{r_{l}}^{m_{l} n_{l}}$ are the corresponding modal amplitudes of the $\left(m_{l}, n_{l}\right)$ mode inside the lth cavity. $\varphi_{l}^{m_{l} l_{l}}\left(y_{l}, z_{l}\right)$ is the eigenfunction of $\left(m_{l}, n_{l}\right)$ mode in the $l$ th cavity and for a rectangular cavity with rigid walls, it is given by

$$
\varphi_{l}^{m_{l} n_{l}}\left(y_{l}, z_{l}\right)=\cos \left(\frac{m_{l} \pi}{L_{y}^{l}} y_{l}\right) \cos \left(\frac{n_{l} \pi}{L_{z}^{l}} z_{l}\right)
$$

where $L_{y}^{l}$ and $L_{z}^{l}$ are the width and height of the lth cavity, respectively.

As shown by Eq. (1), the sound field inside each cavity is expressed by the superposition of $p_{l}^{i}$ and $p_{l}^{r}$, whose propagating directions will be coincident to $x$ axis if $P_{i_{l}}^{m_{l} n_{l}}$ and $P_{r_{l}}^{m_{l} n_{l}}$ are real numbers. However, $P_{i_{l}}^{m_{l} n_{l}}$ and $P_{r_{l}}^{m_{l} n_{l}}$ are mostly complex numbers, which means $p_{l}^{i}$ and $p_{l}^{r}$ will propagate along $x$ axis with an included angle. As a summation of $p_{l}^{i}$ and $p_{l}^{r}$, the entire sound propagating direction inside each cavity could be expressed to be along $x$ direction (e.g., inside cavity $\mathrm{V}_{1}$ ) or $z$ direction (e.g., inside cavity $\mathrm{V}_{3}$ ).

The wave numbers $k_{x_{l}}^{m_{l} n_{l}}, k_{y_{l}}^{m_{l}}, k_{z_{l}}^{n_{l}}$, and $k$ are related by

$$
\begin{aligned}
& k^{2}=(\omega / c)^{2}=\left(k_{x_{l}}^{m_{l} n_{l}}\right)^{2}+\left(k_{y_{l}}^{m_{l}}\right)^{2}+\left(k_{z_{l}}^{n_{l}}\right)^{2}, \\
& k_{y_{l}}^{m_{l}}=\frac{m_{l} \pi}{L_{y}^{l}}, k_{z_{l}}^{n_{l}}=\frac{n_{l} \pi}{L_{z}^{l}},
\end{aligned}
$$

where $\omega$ is the angular frequency, $c$ is the speed of sound

The unknown modal amplitudes $P_{i_{l}}^{m_{l} n_{l}}$ and $P_{r_{l}}^{m_{l} n_{l}}$ are determined by the incident wave condition, the rigid boundary condition at plane $\mathrm{F}$ and the continuity conditions of coupled cavities. ${ }^{21}$ For the problem presented here, the incidence condition is

$$
\left.\left(p_{1}^{i}=P_{0}\right)\right|_{S_{1}}, \quad \text { at } \quad x_{1}=0,
$$

where $\left.\right|_{S}$ denotes that the expression is valid on $S . P_{0}$ is the amplitude of the incident plane wave. $S_{1}$ denotes the cross section of cavity $\mathrm{V}_{1}$.

The rigid boundary condition at plane $\mathrm{F}$ is

$$
\left.\left(v_{5}^{i}+v_{5}^{r}=0\right)\right|_{S_{5}}, \quad \text { at } \quad x_{5}=L_{x}^{5},
$$

where $S_{5}$ denotes the cross section of cavity $\mathrm{V}_{5} . v_{l}^{i}$ and $v_{l}^{r}$ (here $l=5$ ) are the normal velocities in positive and negative $x$ directions in the lth cavity, respectively, and they can be derived from Eq. (1) by using $v=-\frac{1}{\rho} \int \frac{\partial p}{\partial x} d t$ as

$$
v_{l}^{i}=\frac{1}{\rho c} \sum_{m_{l}=0}^{\infty} \sum_{n_{l}=0}^{\infty} K_{x_{l}}^{m_{l} n_{l}} P_{i_{l}}^{m_{l} n_{l}} \varphi_{l}^{m_{l} n_{l}}\left(y_{l}, z_{l}\right) \exp \left(-j k_{x_{l}}^{m_{l} n_{l}} x_{l}\right)
$$


$v_{l}^{r}=-\frac{1}{\rho c} \sum_{m_{l}=0}^{\infty} \sum_{n_{l}=0}^{\infty} K_{x_{l}}^{m_{l} n_{l}} P_{r_{l}}^{m_{l} n_{l}} \varphi_{l}^{m_{l} n_{l}}\left(y_{l}, z_{l}\right) \exp \left(j k_{x_{l}}^{m_{l} n_{l}} x_{l}\right)$,

where $K_{x_{l}}^{m_{l} n_{l}}=k_{x_{l}}^{m_{l} n_{l}} / k$ is the dimensionless wave number, and $\rho$ is the density of air. Similar to Eq. (1), the normal velocity inside each cavity can also be expressed by the superposition of $v_{l}^{i}$ and $v_{l}^{r}$ which respectively denote the propagation along the positive and negative $x$ directions.

At the interfaces of adjacent cavities, the continuity conditions of sound pressure and normal velocity are

$$
\begin{aligned}
& \left.\left(p_{1}^{i}+p_{1}^{r}=p_{2}^{i}+p_{2}^{r}\right)\right|_{S_{12},}, \\
& \left(v_{1}^{i}+v_{1}^{r}\right)=\left.\left(v_{2}^{i}+v_{2}^{r}\right)\right|_{S_{12}}, \\
& \left.\left(p_{2}^{i}+p_{2}^{r}=p_{3}^{i}+p_{3}^{r}\right)\right|_{S_{23}}, \\
& \left(v_{2}^{i}+v_{2}^{r}\right)=\left.\left(v_{3}^{i}+v_{3}^{r}\right)\right|_{S_{23}}, \\
& \left.\left(p_{3}^{i}+p_{3}^{r}=p_{4}^{i}+p_{4}^{r}\right)\right|_{S_{34}}, \\
& \left(v_{3}^{i}+v_{3}^{r}\right)=\left.\left(v_{4}^{i}+v_{4}^{r}\right)\right|_{S_{34}}, \\
& \left.\left(p_{4}^{i}+p_{4}^{r}=p_{5}^{i}+p_{5}^{r}\right)\right|_{S_{45}}, \\
& \left(v_{4}^{i}+v_{4}^{r}\right)=\left.\left(v_{5}^{i}+v_{5}^{r}\right)\right|_{S_{45}},
\end{aligned}
$$

where $S_{i j}$ denotes the interface of cavity $\mathrm{V}_{i}$ and cavity $\mathrm{V}_{j}$.

To solve the problem, the summation of modes is truncated to be finite, following a rule that the modes of each cavity are chosen to be those whose eigenfrequencies are below a frequency $\left(f_{u}\right)$, which is seven times the considered frequency. The total number of the modes considered in all the five cavities is

$$
Q_{0}=Q_{1}+Q_{2}+Q_{3}+Q_{4}+Q_{5},
$$

where $Q_{1}, Q_{2}, Q_{3}, Q_{4}$, and $Q_{5}$ are the number of modes considered in each cavity, respectively.

The orthogonality of the eigenfunctions is applied to obtain a set of linear equations from Eqs. (4) and (5), and Eqs. (7) to (10) (see Appendix) and they can be written in a matrix form

$$
\mathbf{A P}=\mathbf{C},
$$

where

$$
\begin{aligned}
& \mathbf{P}=\left[\mathbf{P}_{i_{1}, x_{1}=0}^{m_{1} n_{1}}, \mathbf{P}_{r_{1}, x_{1}=0}^{m_{1} n_{1}}, \mathbf{P}_{i_{2}, x_{2}=0}^{m_{2} n_{2}}, \mathbf{P}_{r_{2}, x_{2}=0}^{m_{2} n_{2}}, \mathbf{P}_{i_{3}, x_{3}=0}^{m_{3} n_{3}},\right. \\
& \left.\mathbf{P}_{r_{3}, x_{3}=0}^{m_{3} n_{3}}, \mathbf{P}_{i_{4}, x_{4}=0}^{m_{4} n_{4}}, \mathbf{P}_{r_{4}, x_{4}=0}^{m_{4} n_{4}}, \mathbf{P}_{i_{5}, x_{5}=0}^{m_{5} n_{5}}, \mathbf{P}_{r_{5}, x_{5}=0}^{m_{5} n_{5}}\right]^{T}
\end{aligned}
$$

is a $2 Q_{0}$ vector of the unknown modal amplitudes, and the superscript $T$ denotes transposition

$$
\begin{aligned}
& \mathbf{C}=\left[P_{0,1}^{00}, \ldots, P_{0,1}^{M_{1}^{\prime} N_{1}^{\prime}}, \mathbf{0}, \ldots, \mathbf{0}\right]^{T}, \\
& P_{0,1}^{m_{1}^{\prime} n_{1}^{\prime}}=P_{0}\left\langle\varphi_{1}^{m_{1}^{\prime} n_{1}^{\prime}}\left(y_{1}, z_{1}\right)\right\rangle_{S_{1}}
\end{aligned}
$$

is a $2 Q_{0}$ vector of solutions. In Eq. (14) \langle\rangle$_{s}$ denotes integration of the expression inside the parenthesis over the area indicated with the subscript. $M^{\prime}$ and $N^{\prime}$ are the maximums of $m$ and $n$, respectively. $\mathbf{A}$ is a $2 Q_{0} \times 2 Q_{0}$ matrix of coefficients and its expression is given in Appendix.

If the amplitude of the incident plane wave, $P_{0}$, is prescribed, the unknown modal coefficients can be determined. Equation (12) has a unique solution, provided that $\mathbf{A}$ is not singular and has an inverse, and thus

$$
\mathbf{P}=\mathbf{A}^{-1} \mathbf{C}
$$

By solving Eq. (12), one can obtain the sound pressure in each cavity by substituting $P_{i_{l}}^{m_{l} n_{l}}$ and $P_{r_{l}}^{m_{l} n_{l}}$ into Eq. (1).

\section{Secondary sound field}

To implement ANC, control sources are placed inside the staggered window system to produce the secondary sound field. As the dimensions of commonly used loudspeakers are small compared with the wavelength of low frequency sound concerned here, the control sources are regarded as point sources for the sake of convenience. The determination of the sound fields inside each cavity with the point source excitation is similar to that with plane wave incidence. Without plane wave incidence, the sound field inside cavity $\mathrm{V}_{1}$ solely consists of propagation in the negative $x$ direction,

$$
p_{1}^{S}=p_{1}^{r}=\sum_{m_{1}=0}^{\infty} \sum_{n_{1}=0}^{\infty} \varphi_{1}^{m_{1} n_{1}}\left(y_{1}, z_{1}\right) P_{r_{1}}^{m_{1} n_{1}} \exp \left(j k_{x_{1}}^{m_{1} n_{1}} x_{1}\right)
$$

To produce a secondary sound field, a point source is placed inside cavity $\mathrm{V}_{3}$ at $\left(x_{3}^{S}, y_{3}^{S}, z_{3}^{S}\right)$, and the sound field inside $\mathrm{V}_{3}$ can be written as ${ }^{22}$

$$
\begin{aligned}
p_{3}= & p_{3}^{P}+p_{3}^{q}=\left(p_{3}^{i}+p_{3}^{r}\right)+p_{3}^{q} \\
= & \sum_{m_{3}=0}^{\infty} \sum_{n_{3}=0}^{\infty} \varphi_{3}^{m_{3} n_{3}}\left(y_{3}, z_{3}\right)\left[P_{i_{3}}^{m_{3} n_{3}} \exp \left(-j k_{x_{3}}^{m_{3} n_{3}} x_{3}\right)\right. \\
& \left.+P_{r_{3}}^{m_{3} n_{3}} \exp \left(j k_{x_{3}}^{m_{3} n_{3}} x_{3}\right)\right]+\frac{-j \omega \rho q_{c}}{2 L_{y}^{3} L_{z}^{3}} \\
& \times \sum_{m_{3}=0}^{\infty} \sum_{n_{3}=0}^{\infty} \frac{\varphi_{3}^{m_{3} n_{3}}\left(y_{3}, z_{3}\right) \varphi_{3}^{m_{3} n_{3}}\left(y_{3}^{S}, z_{3}^{S}\right)}{k_{x_{3}}^{m_{3} n_{3}} D_{y z}\left(m_{3}, n_{3}\right)} \\
& \times \exp \left(-j k_{x_{3}}^{m_{3} n_{3}}\left|x_{3}-x_{3}^{S}\right|\right),
\end{aligned}
$$

where $q_{c}$ is the volume velocity of the point source, and

$$
D_{y z}\left(m_{3}, n_{3}\right)=\frac{1}{D_{y}\left(m_{3}\right) D_{z}\left(n_{3}\right)},
$$

where $D_{y}\left(m_{3}\right)=1$ for $m_{3}=0$, and $D_{y}\left(m_{3}\right)=2$ for $m_{3} \neq 0$, and the same for $D_{z}\left(n_{3}\right)$.

The term $p_{3}^{P}$ models sound propagating from plane $\mathrm{C}$ and plane $\mathrm{D}$ and the expression is the same as the one with plane wave incidence. The term $p_{3}^{q}$ models sound propagating from the point source and it is taken as the pressure with a point source set in an infinite rectangular duct. ${ }^{21}$

The expressions for the sound fields inside cavity $V_{2}$, $\mathrm{V}_{4}$, and $\mathrm{V}_{5}$ with the point source excitation are the same as those with plane wave incidence, as expressed by Eq. (1). To 
solve the secondary sound field, there are nine groups of boundary condition and continuity conditions, given by Eqs. (5), (7), (8), (9), and (10), respectively. The procedures of solving the secondary sound field are similar to those of solving the primary sound field, so they are not presented here.

\section{Active noise control}

The sum of the squared sound pressures at the error sensors is selected as the cost function

$$
J=\sum_{i=1}^{N_{e}}\left|p_{e i}\right|^{2}+\beta \mathbf{q}_{s}^{H} \mathbf{q}_{s},
$$

where $p_{e i}$ is the sound pressure at the $i$ th error sensor. $N_{e}$ is the number of the error sensors. $\beta$ is a real number, which is used to determine the weighting for the control effort. The superscript $H$ denotes Hermitian transpose of a matrix. $\mathbf{q}_{s}=\left[q_{1}, q_{2}, \ldots, q_{N_{c}}\right]^{T}$ is the vector of the strengths of the control sources and the optimum value is ${ }^{23}$

$$
\mathbf{q}_{s s}=-\left(\mathbf{Z}_{s e}^{H} \mathbf{Z}_{s e}+\beta \mathbf{I}\right)^{-1} \mathbf{Z}_{s e}^{H} \mathbf{P}_{p e},
$$

where $N_{c}$ is the number of the control sources. $\mathbf{P}_{p e}=\left[p_{p e_{1}}, p_{p e_{2}}, \ldots, p_{p e_{N_{c}}}\right]^{T}$ is the vector of the sound pressures at the error sensors due to the primary plane wave. $\mathbf{Z}_{s e}$ is the matrix of the transfer functions from the control sources to the error sensors and can be expressed as

$$
\mathbf{Z}_{s e}=\left[\begin{array}{ccc}
Z\left(\mathbf{r}_{s_{1} e_{1}}\right) & \cdots & Z\left(\mathbf{r}_{s_{N c} e_{1}}\right) \\
\vdots & \ddots & \vdots \\
Z\left(\mathbf{r}_{s_{1} e_{N e}}\right) & \cdots & Z\left(\mathbf{r}_{s_{N c} e_{N e}}\right)
\end{array}\right]
$$

where $\mathbf{r}_{s_{i} e_{j}}$ and $Z\left(\mathbf{r}_{s_{i} e_{j}}\right)$ are the distance vector and transfer function from the $i$ th secondary source to the $j$ th error sensor, respectively.

The control performance is defined as the EA of the averaged SPL at the observation points due to ANC, namely, the difference between the averaged SPLat the observation points with and without active control

$$
E A=S P L_{\text {without }}-S P L_{\text {with }},
$$

and the averaged SPLis defined as

$$
S P L_{\text {averaged }}=10 \log _{10} \frac{\sum_{i=1}^{N_{o}}\left|p_{o i}\right|^{2}}{N_{o} p_{\text {ref }}^{2}},
$$

where $p_{o i}$ is the sound pressure at the $i$ th observation point. $N_{o}$ is the number of the observation points. $p_{\text {ref }}=20 \times 10^{-6} \mathrm{~Pa}$ is the reference pressure.

To implement the active noise control simulations, the primary sound field at the error sensor $\mathbf{P}_{p e}$ and the matrix $\mathbf{Z}_{s e}$ are calculated with plane wave incidence (e.g., $P_{0}=1$ ) and point source excitation (e.g., the volume strength is $0.001 \mathrm{~m}^{3} / \mathrm{s}$ ), respectively, by using the analytical model
TABLE I. Geometrical dimensions of the model used for validations.

\begin{tabular}{lccc}
\hline \hline & $L_{x}(\mathrm{~m})$ & $L_{y}(\mathrm{~m})$ & $L_{z}(\mathrm{~m})$ \\
\hline Cavity $\mathrm{V}_{1}$ & - & 4.25 & 1.00 \\
Cavity $\mathrm{V}_{2}$ & 0.01 & 0.85 & 0.20 \\
${\text { Cavity } \mathrm{V}_{3}}_{\text {Cavity } \mathrm{V}_{4}}$ & 0.29 & 0.85 & 1.27 \\
Cavity $\mathrm{V}_{5}$ & 0.01 & 0.85 & 0.20 \\
\hline \hline
\end{tabular}

developed above. Then substitute $\mathbf{P}_{p e}$ and $\mathbf{Z}_{s e}$ into Eq. (20), the optimum volume strength of secondary sources, $\mathbf{q}_{\text {os }}$, can be calculated, with which the analytical model is again used to calculate the optimized secondary sound field. Finally, the total residual sound field is obtained by adding the primary sound field and the optimized secondary sound field.

In this paper, $N_{c}=N_{e}=1,2$, and $\beta$ is adjusted so that the noise reduction at the error sensors is not greater than 20 $\mathrm{dB}$ as that could be achieved in practical situations.

\section{NUMERICAL SIMULATION AND DISCUSSION}

\section{A. Validation of the proposed analytical models}

FEM is employed to validate the proposed analytical model, where a commercial software COMSOL is used. ${ }^{24}$ In the FEM model, the five coupled cavities shown in Fig. 1(b) are created with the geometrical dimensions shown in Table I, where rigid boundary conditions are used for the walls of the room and the window and plane wave radiation condition is used at plane A. The maximum length of mesh elements is chosen to be $1 / 10$ of the wavelength in the FEM model, in order to guarantee sufficient calculation accuracy. It can be seen from Table I that $L_{y}^{1}$ and $L_{z}^{1}$ are respectively selected as five times of $L_{y}^{2}$ and $L_{z}^{2}$, which are sufficiently large to simulate the SSO placed in an infinite baffle. For the
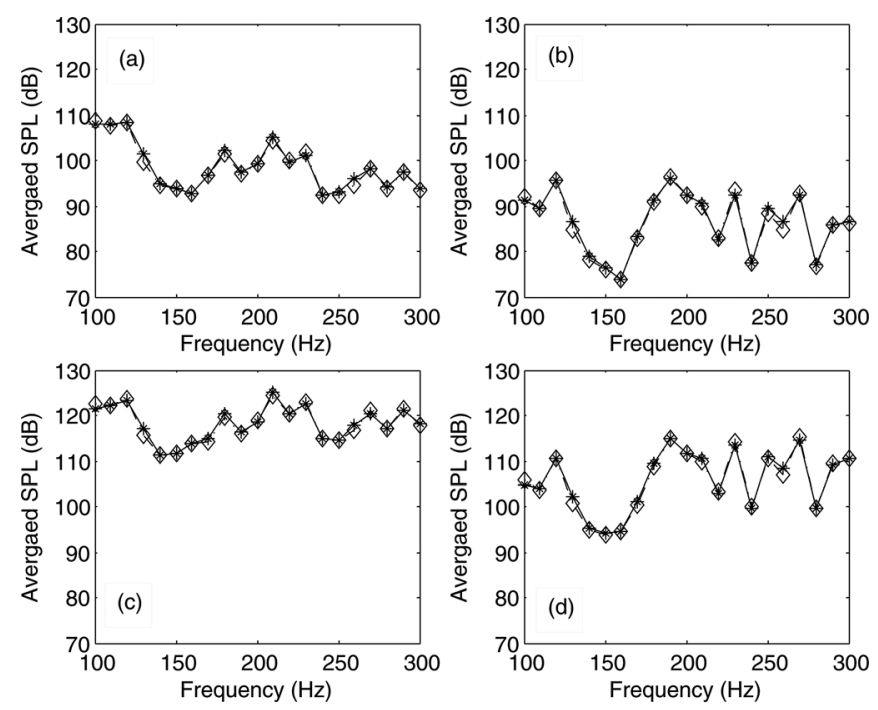

* * Proposed model $\cdot-\diamond-\cdot$ FEM model

FIG. 2. Comparisons of the averaged SPLs obtained by the proposed model and the FEM model. (a) Inside the staggered window system with plane wave incidence. (b) Inside the accompanying room with plane wave incidence. (c) Inside the staggered window system with point source excitation. (d) Inside the accompanying room with point source excitation. 


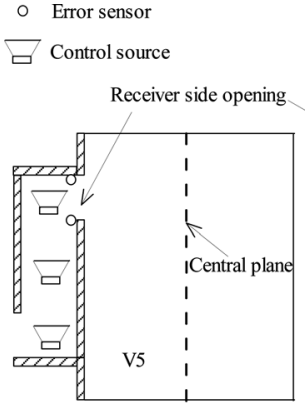

(a)

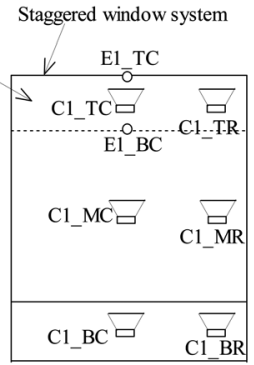

(b)

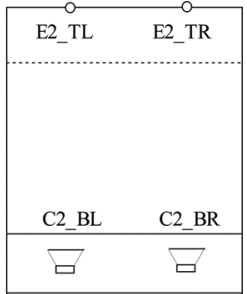

(c)
FIG. 3. Sketch of the configurations of the ANC system used in the numerical simulations. (a) Cross section. (b) Front view of a single channel ANC system. (c) Front view of a double channel ANC system.

secondary sound field, a point source is placed inside cavity $\mathrm{V}_{3}$ and its coordinates are $(0.140,0.425,0.100 \mathrm{~m})$.

Figures 2(a) and 2(b) respectively compare the averaged SPLs in the staggered window system and in the room with the plane wave incidence, where the discrepancies are mostly within $1 \mathrm{~dB}$. Figures 2(c) and 2(d) respectively show comparison of the averaged SPLs in the staggered window system and in the room with a secondary point source excitation. The results agree well and the discrepancies are between 0.01 and $1.39 \mathrm{~dB}$, showing the validity of the proposed analytical model of the secondary sound field.

\section{B. Feasibility of using active noise control techniques}

The primary sound field is established by a plane wave incident from cavity $\mathrm{V}_{1}$. The control sources are point sources placed inside cavity $\mathrm{V}_{3}$ and the error sensors are located around the RSO. The observation points are evenly distributed inside cavity $\mathrm{V}_{5}$ and the spacing between adjacent observation points is $0.05 \mathrm{~m}$, which is sufficient for noise reduction performance evaluation under $600 \mathrm{~Hz}$.

\section{Single channel system}

With $N_{c}=N_{e}=1$, EA are evaluated with six potential locations of the control source and two potential locations of the error sensor. The arrangements are illustrated in Figs. 3(a) and 3(b), where the control source is placed either at the bottom (abbreviated by letter B), middle (M) or top (T) of the staggered window system along the vertical direc- tion and there are two horizontal positions for each vertical location, at right $(\mathrm{R})$ and center $(\mathrm{C})$ of the staggered window system, respectively. The error sensor $(\mathrm{E})$ is placed either at the bottom or top horizontal edge of the RSO and it is at the central horizontal position. The locations of control source and error sensor are indicated in Fig. 3(b) and the corresponding coordinates are listed in Table II.

The results of EA obtained with the control source placed at the vertical right (i.e., C1_BR, C1_MR and C1_TR) or central side (i.e., C1_BC, C1_MC and C1_TC) of the staggered window system are shown in Fig. 4. When the control source is at the horizontal right position, EA is around $20 \mathrm{~dB}$ from 100 to $200 \mathrm{~Hz}$ and it decreases significantly to less than $20 \mathrm{~dB}$ and fluctuates with frequency at 210-600 Hz, as shown in Figs. 4(a) and 4(c). When the control source is placed at the bottom central position of the staggered window system, the ANC system is effective from 100 to $390 \mathrm{~Hz}$ and an EA around $20 \mathrm{~dB}$ can always be achieved, as shown in Fig. 4(b). When the control source is placed at the middle central position of the staggered window system, the frequency of effective control ranges from 100 to $340 \mathrm{~Hz}$, as shown in Fig. 4(d), which is narrower than that when the control source is at the bottom central position. When the control source is located at the top of the staggered window system, EA varies significantly with frequency, as shown in Figs. 4(e) and 4(f), where the performance of the ANC system is not good, and the noise even increases at some frequency ranges, i.e., with negative EA values. It seems that the optimal arrangement for a single channel system is placing the control source at the bottom central position of the staggered window system, with which noise from 100 to $390 \mathrm{~Hz}$ could be effectively controlled in the room, and the difference between the EAs obtained with the error sensor placed at position E1_TC and E1_BC is insignificant.

The main mechanism for effective active control is sound field matching, namely, the secondary sound field in the room has a similar shape to the primary sound field. Figure 5 shows the SPL distributions at $250 \mathrm{~Hz}$ and at the central $y_{5}-z_{5}$ plane $\left(x_{5}=1.5 \mathrm{~m}\right)$ of the room with the primary plane wave excitation or with the optimized control source excitation when it is at $\mathrm{C} 1 \_\mathrm{BR}$ and at $\mathrm{C} 1 \_\mathrm{BC}$, respectively, as illustrated in Fig. 3(a). It can be seen that the secondary sound field with the control source at C1_BC matches well with the primary sound field, while if the control source is at C1_BR, the secondary sound field is different from the

TABLE II. Coordinates of the control source and the error sensor shown in Fig. 3(b) and the corresponding extra attenuations at 150, 250, 350, and 450 Hz, which are presented as: with error sensor at (the top edge)/(the bottom edge). The terms $\mathrm{C} 1$ denotes 1 control source; the letters B, M and T are, respectively, the abbreviation of bottom, middle and top; the letters $\mathrm{R}$ and $\mathrm{C}$ are, respectively, the abbreviation of right and center; the letter E denotes error sensor.

\begin{tabular}{|c|c|c|c|c|c|c|c|}
\hline & $x_{3}(\mathrm{~m})$ & $y_{3}(\mathrm{~m})$ & $z_{3}(\mathrm{~m})$ & $150 \mathrm{~Hz}$ & $250 \mathrm{~Hz}$ & $350 \mathrm{~Hz}$ & $450 \mathrm{~Hz}$ \\
\hline C1_BR & 0.1400 & 0.2125 & 0.1000 & $19.4 / 19.7$ & $-6.0 /-6.0$ & $-5.8 /-5.9$ & $8.2 / 9.6$ \\
\hline C1_BC & 0.1400 & 0.4250 & 0.1000 & $20.4 / 19.8$ & 20.0/19.8 & $16.3 / 31.4$ & $8.8 / 12.3$ \\
\hline C1_MR & 0.1400 & 0.2125 & 0.6350 & $14.0 / 14.2$ & $-13.2 /-13.2$ & $-6.1 /-6.3$ & $1.4 /-0.6$ \\
\hline C1_MC & 0.1400 & 0.4250 & 0.6350 & $21.0 / 21.9$ & $22.1 / 27.8$ & $1.8 / 5.4$ & $9.3 / 12.8$ \\
\hline C1_TR & 0.1400 & 0.2125 & 1.1700 & $0.3 /-4.6$ & $-4.2 / 0.5$ & $1.9 / 1.9$ & $5.9 / 5.9$ \\
\hline C1_TC & 0.1400 & 0.4250 & 1.1700 & $5.1 / 12.3$ & $-2.8 / 13.0$ & $0.3 / 1.2$ & $8.8 /-18.0$ \\
\hline E1_BC & 0.2800 & 0.4250 & 1.0700 & - & - & - & - \\
\hline E1_TC & 0.2800 & 0.4250 & 1.2700 & - & - & - & - \\
\hline
\end{tabular}



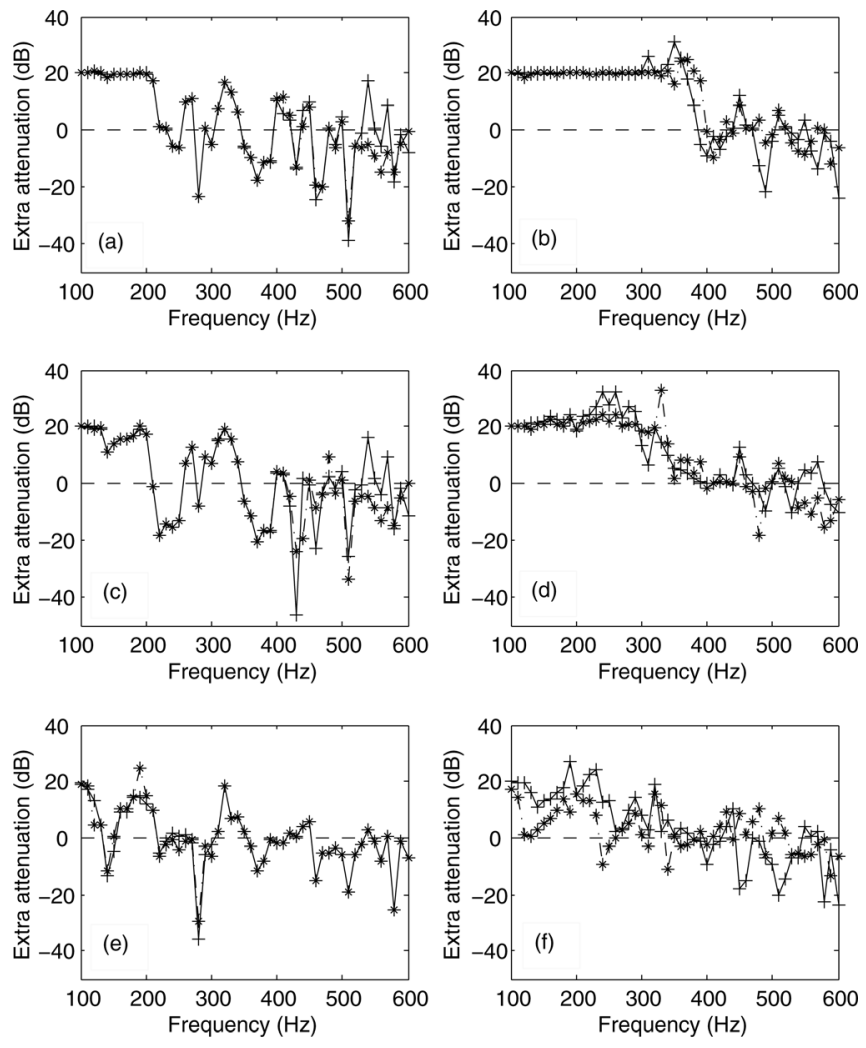

*- Error sensor is palced at the top edge of the receiver opening 1 Error sensor is palced at the bottom edge of the receiver opening

FIG. 4. Extra attenuations obtained when a single channel ANC system is used, with the control source placed at the (a) bottom right position, (b) bottom central position, (c) middle right position, (d) middle central position, (e) top right position, (f) top central position.

primary sound field. Further simulations show that this conclusion is not only valid at the central plane, but also valid at other planes. Consequently, when the control source is at C1_BC, an effective control is achieved, as can be seen in Fig. 4(b), whereas if the control source is at C1_BR, no effective control can be achieved, as can be seen in Fig. 4(a). Moreover, when the primary sound field is perfectly controlled, the EA is approximately equivalent to the noise reduction at the error sensor.

\section{Double channel system}

The performance of multi channel ANC system is investigated with a double channel system with the optimal arrangements, namely, the control sources are evenly placed at the bottom position of the staggered window system and the error sensors are located at the top edge of the RSO, as shown in Fig. 3(c). The coordinates of the control sources and error sensors are listed in Table III.

Figure 6 shows the EAs obtained with the double channel ANC system. It can be seen that the frequency range of effective control is from 100 to $410 \mathrm{~Hz}$. From 420 to $600 \mathrm{~Hz}$, although EA varies significantly with frequency, positive EA can still be obtained at most frequencies, which is better than those with a single channel system. The reason for the wider frequency range of effective control which could be achieved inside the room with a double channel system is that the sound (a)
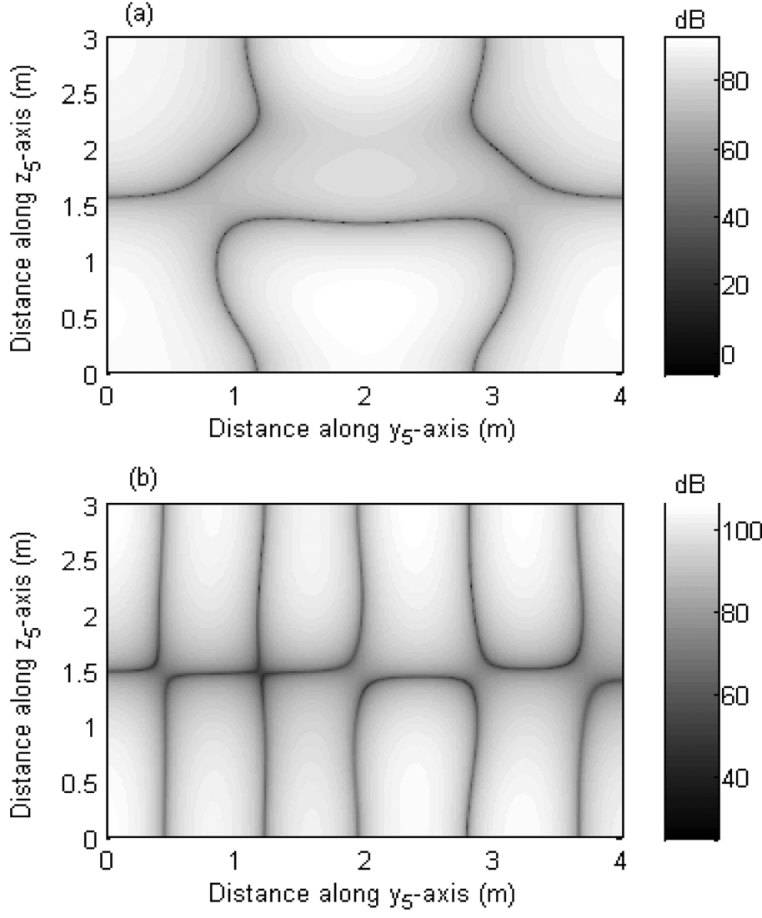

(c)

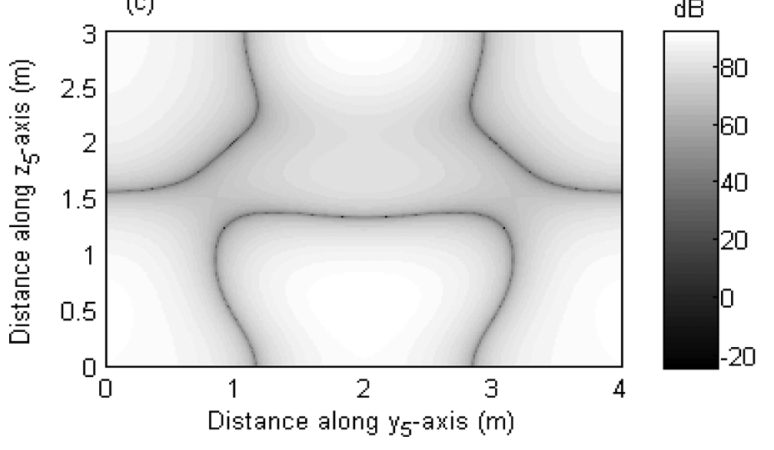

FIG. 5. SPL distributions at the central $y_{5}-z_{5}$ plane $\left(x_{5}=1.5 \mathrm{~m}\right)$ of the room at $250 \mathrm{~Hz}$. (a) Primary plane wave excitation. (b) Control source excitation when it is at the bottom right position. (c) Control source excitation when it is at the bottom central position.

field at the RSO could be better matched at relatively high frequency when two error sensors are used, and this is similar to that in free space, where two or three error sensors should be arranged within a wavelength to guarantee effective control. ${ }^{25}$ It can also be observed that a negative EA is obtained at frequencies around $600 \mathrm{~Hz}$. A possible reason is that as the frequency increases, the sound field at the RSO varies

TABLE III. Coordinates of the control sources and the error sensors of a double channel ANC system shown in Fig. 3(c) and the corresponding extra attenuations at 150,250,350, and $450 \mathrm{~Hz}$. The terms $\mathrm{C} 2$ denotes two control sources; the letters B is the abbreviation of bottom; the letters R, C, and L are the abbreviation of right, center and left, respectively; the letter $\mathrm{E}$ denotes error sensor.

\begin{tabular}{lccccccc}
\hline \hline & $x_{3}(\mathrm{~m})$ & $y_{3}(\mathrm{~m})$ & $z_{3}(\mathrm{~m})$ & $150 \mathrm{~Hz}$ & $250 \mathrm{~Hz}$ & $350 \mathrm{~Hz}$ & $450 \mathrm{~Hz}$ \\
\hline C2_BR & 0.1400 & 0.2125 & 0.1000 & 20.3 & 20.0 & 19.8 & 4.2 \\
C2_BL & 0.1400 & 0.6375 & 0.1000 & & & & \\
E2_TR & 0.2800 & 0.2125 & 1.2700 & - & - & - & - \\
E2_TL & 0.2800 & 0.6375 & 1.2700 & & & & \\
\hline
\end{tabular}




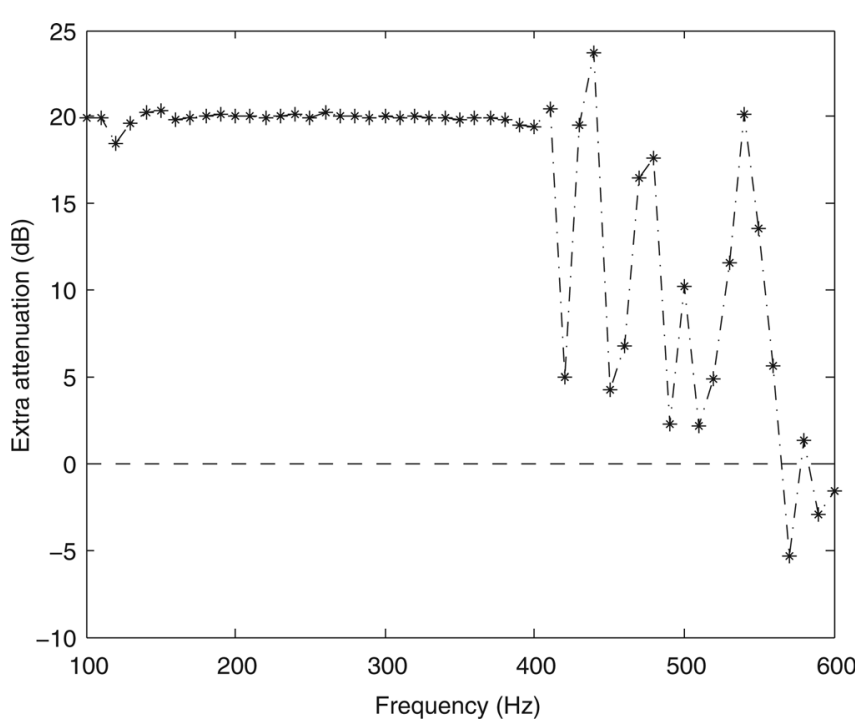

FIG. 6. Extra attenuations obtained when a double channel ANC system is used with the control sources placed at the vertical bottom position and the error sensors placed at the top edge of the receiver side opening, as shown in Fig. 3(c).

significantly with distance and the error sensors might be placed at the troughs of the sound field, resulting in the sound pressures at the other places of the RSO increases, despite that the noises are reduced at the error sensors.

\section{EXPERIMENTAL VALIDATION}

\section{A. Experimental setup}

The experiments were carried out in a large anechoic chamber. Due to the size of secondary sources and the volume of the entire experimental setup, a 1:2 scale model of the one used in the numerical simulations was used for convenience. The dimensions and frequencies below refer to model scale, except where indicated. Figure 7 shows the model window system and the room with a single channel ANC system schematically. The panels of the window are made of acrylic glass with a density of $\rho_{p}=1220 \mathrm{~kg} / \mathrm{m}^{3}$ and a thickness of about $10 \mathrm{~mm}$. The dimensions of the panels are about $0.42 \mathrm{~m} \times 0.53 \mathrm{~m}$ and the space between the two panels is about $0.15 \mathrm{~m}$. The inner dimensions of the room are $1.90 \mathrm{~m} \times 1.22 \mathrm{~m} \times 1.26 \mathrm{~m}$ and the room and the frames of the window are made of $18 \mathrm{~mm}$ thick wooden boards. In the room, the walls are covered by about $5 \mathrm{~cm}$ thick absorbing materials to reduce reverberation.

A multi channel adaptive feedforward ANC system embedded with the standard FXLMS algorithm was used in the experiments. Take a single channel ANC system, for example, Fig. 8(a) shows the block diagram of the overall system. A band limited white noise signal, with the frequency ranges from 200 to $1000 \mathrm{~Hz}$, was generated by a Brüel \& Kjær PULSE 3560D analyzer and then amplified before feeding to the primary source. To avoid the pickup of the signal generated by the secondary source, a unidirectional electrets microphone, which could only receive the sound incident from its front side, was used as the reference sensor. It is noted that even if there are sources radiating sounds inside the room (e.g., a TV), the performance of the ANC system will not be affected since these noises cannot be picked up by the reference sensor so they are not coherent with the primary sound field. The error acoustical signal is received by an omnidirectional electrets microphone. A controlling signal was then generated by the controller to drive the secondary source to cancel the primary sound field at the error sensor. Five Brüel \& Kjær 4190 microphones, which were used as the observation sensors, were randomly placed inside the room. The averaged SPL at these five sensors was used to assess the performance of the ANC system. Figure 8(b) shows a photograph of the experimental setup when a single channel system was used.

When the primary source works solely, the averaged SPLat the observation sensors with the SSO and the RSO sealed or open are respectively denoted as $L_{\mathrm{s}}$ and $L_{\mathrm{o}} . L_{\mathrm{o}}-L_{\mathrm{s}}$ is expected to be equivalent to the maximum EA due to the ANC system and the experimental setup shown in Fig. 8. Figure 9 shows the curves of $L_{\mathrm{s}}, L_{\mathrm{o}}$, and the maximum EA. The SPLs of the background noise measured in the room are respectively about $-10 \mathrm{~dB}$ from 200 to $1000 \mathrm{~Hz}$ during the experiments, which means the background SPL can be guaranteed to be $10 \mathrm{~dB}$ lower than the primary sound field even for the lowest SPL around $800 \mathrm{~Hz}$. It can also be seen that

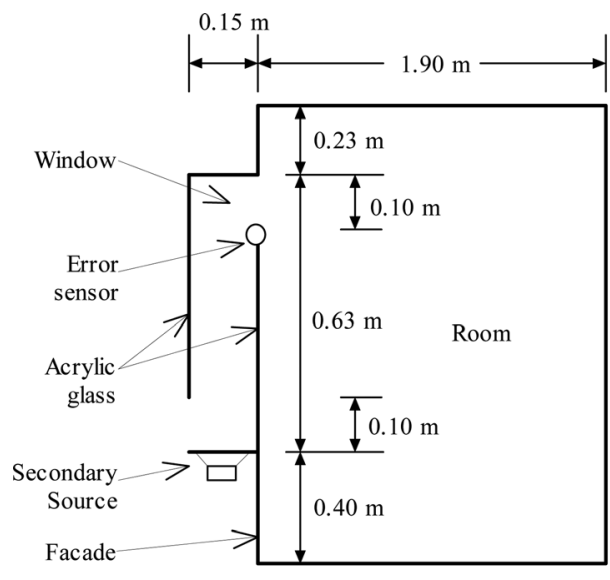

(a)

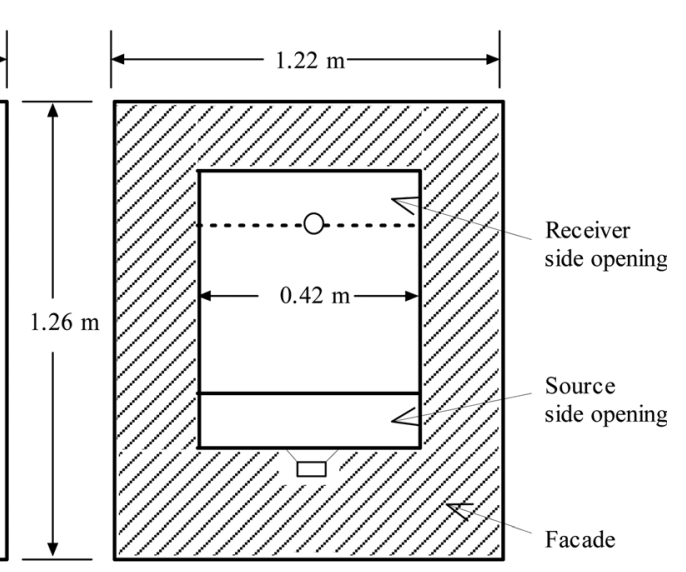

(b) 


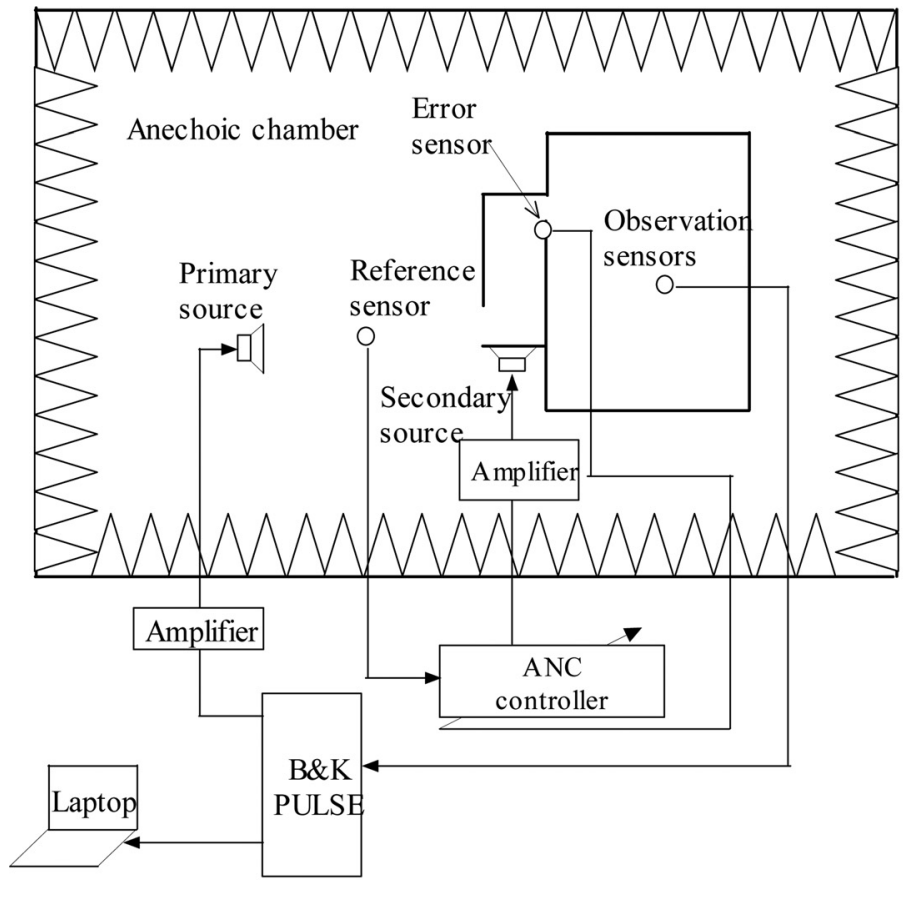

(a)

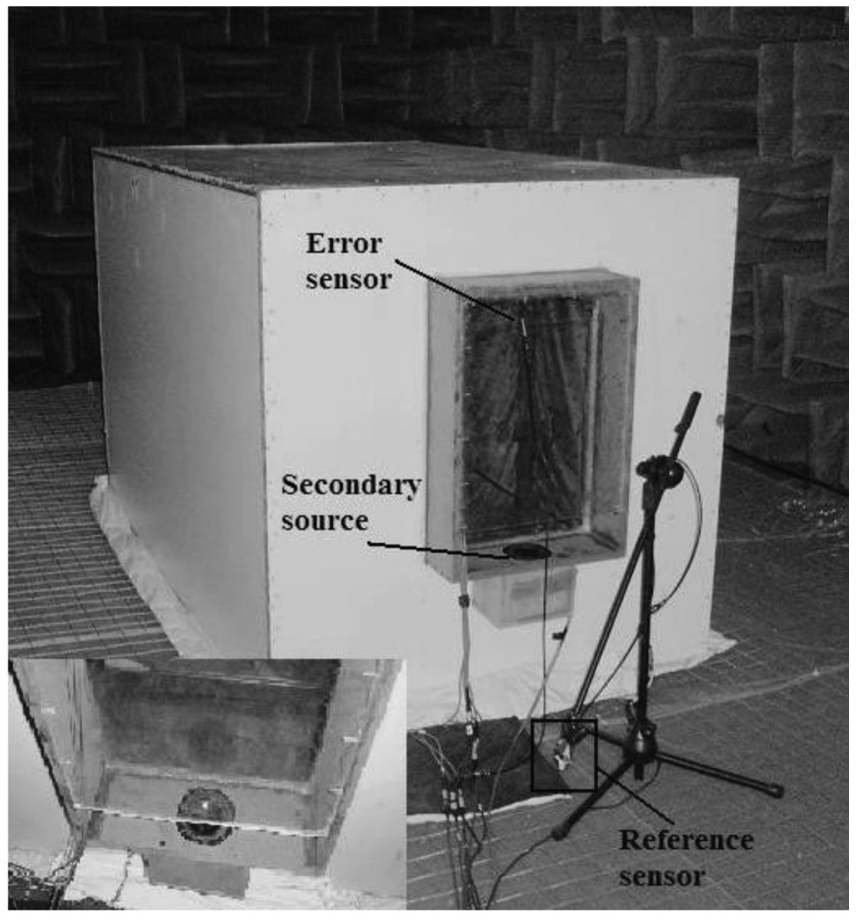

(b)

FIG. 8. Experimental setup of the actively control window system. (a) Sketch map of a single channel ANC system together with the measuring platform. (b) Photograph of (a).

the maximum EA is higher than $10 \mathrm{~dB}$ at most frequencies except a trough exists around $300 \mathrm{~Hz}$.

\section{B. Sources and sensors arrangements}

As shown in Fig. 10(a), the primary source is placed at position N1. The arrangements of the secondary sources of a single channel and a double channel ANC system are shown in Figs. 10(b) and 10(c), where identifiers are marked. "SS" indicates single secondary source and "DS" denotes double

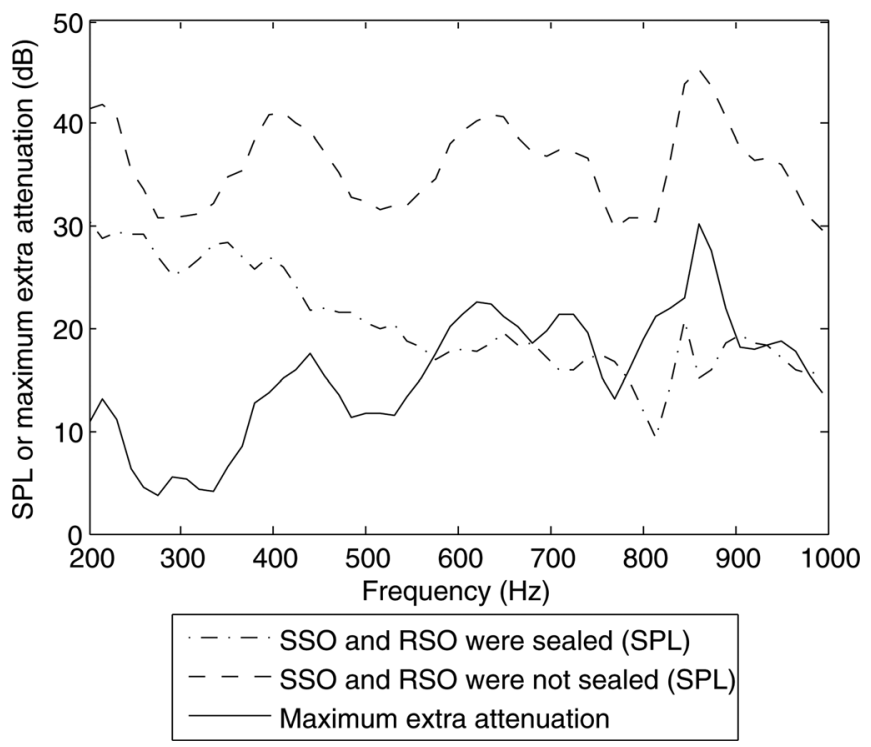

FIG. 9. Averaged SPLs measured when the source side opening and the receiver side opening of the window system were sealed or open. The calculated maximum extra attenuation is also given. secondary sources. The error sensors were placed at the bottom edge of the RSO, respectively, corresponding to position E1_BC for the single channel ANC system and positions E2_BR and E2_BL for the double channel ANC system in the numerical simulations. The distance between the reference sensor and the secondary source is $1 \mathrm{~m}$, which is large enough to guarantee that the causality of the ANC controller is satisfied.

\section{Results and discussions}

The experimental validations were carried out in both frequency domain and time domain.

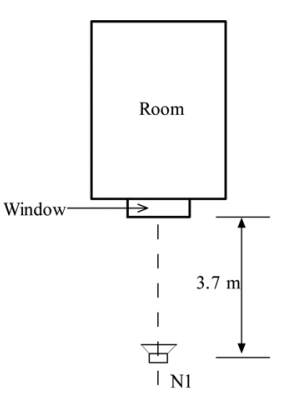

(a)

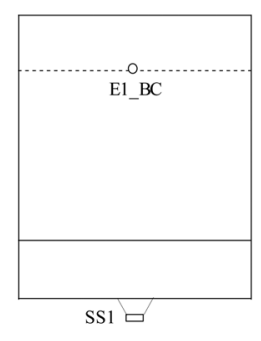

(b)

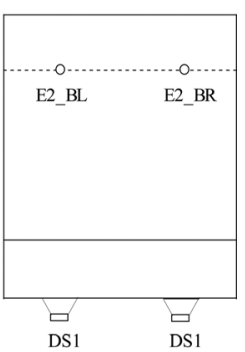

(c)
FIG. 10. Configurations of the primary source, the secondary sources and the error sensors in the experiments. (a) Arrangements of the primary source. (b) Arrangements of the secondary source and the error sensor for the single channel ANC system. (c) Arrangements of the secondary sources and the error sensors for the double channel ANC system. " $\mathrm{N}$ " indicates normal incidence and "O" indicates oblique incidence. "SS" indicates single secondary source and "DS" denotes double secondary sources. 


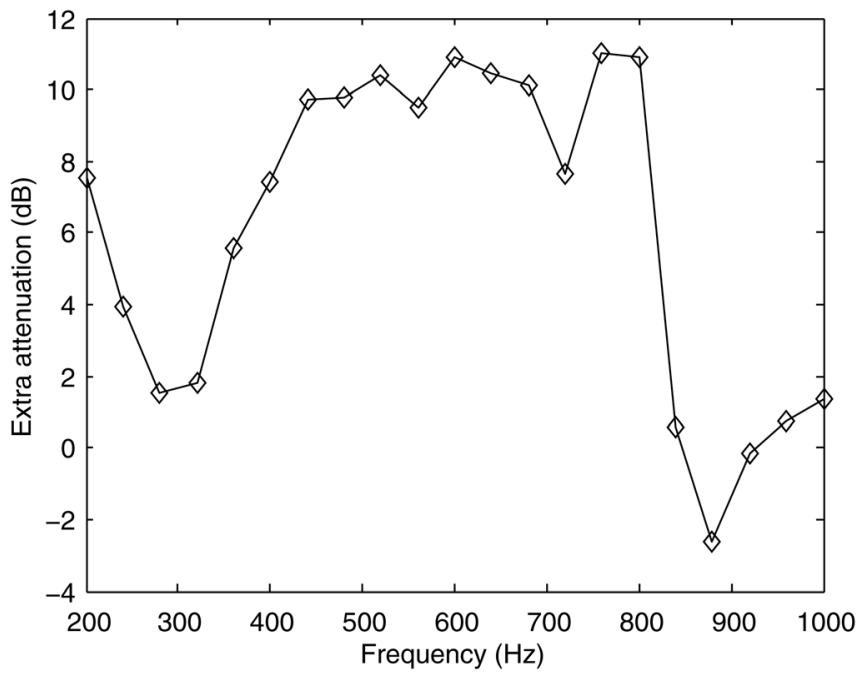

FIG. 11. The extra attenuation at the observation sensors with the noise reduction at the error sensor was controlled to be about $10 \mathrm{~dB}$.

\section{Frequency domain}

When examining the performance of the ANC system in frequency domain, the primary source and the secondary source were placed at position N1 and position SS1, respectively. The frequency of the primary signal ranges from 200 to $1000 \mathrm{~Hz}$ with a step of $40 \mathrm{~Hz}$. Since the maximum EA is larger than $10 \mathrm{~dB}$ at most frequencies, as shown in Fig. 9, and the EA at the observation sensors is expected to be almost the same as the reduction at the error sensor, the noise reduction at the error sensor was controlled to be about 10 $\mathrm{dB}$. Figure 11 shows the corresponding EA at the observation sensors, and it can be noted that the EA is about $10 \mathrm{~dB}$ from 400 to $800 \mathrm{~Hz}$, which is close to the noise reduction at the error sensor. The trough around $300 \mathrm{~Hz}$ is subject to the sound insulation capacity of the experimental setup, as shown in Fig. 9. These results show the validity of the conclusion that the EA at the observation points is almost equivalent to the noise reduction at the error sensor, which agrees well with the results in the numerical simulations.

\section{Time domain}

A single channel and a double channel system with optimized configurations were used to investigate the broadband performance of the ANC system in time domain. Figure 12 shows the averaged SPLat the observation sensors when the ANC system was on or off. It can be seen that the primary sound fields are almost the same and the small differences might be mainly caused by different installation positions of the secondary sources during the experiments. The frequency ranges of effective control are up to about $820 \mathrm{~Hz}$ and $980 \mathrm{~Hz}$ (corresponding to about 410 and $490 \mathrm{~Hz}$ in the numerical simulations) for the single channel and double channel system, respectively, as shown in Figs. 12(a) and 12(b), which are slightly better than those results obtained in the numerical simulations. The EA of the overall SPL $\left(\mathrm{EA}_{\mathrm{t}}\right)$ are respectively 3.8 and $9.6 \mathrm{~dB}$ for the single channel and double channel ANC system. From 200 to $300 \mathrm{~Hz}$, it can be seen that the EA due to the single channel and the double channel ANC system

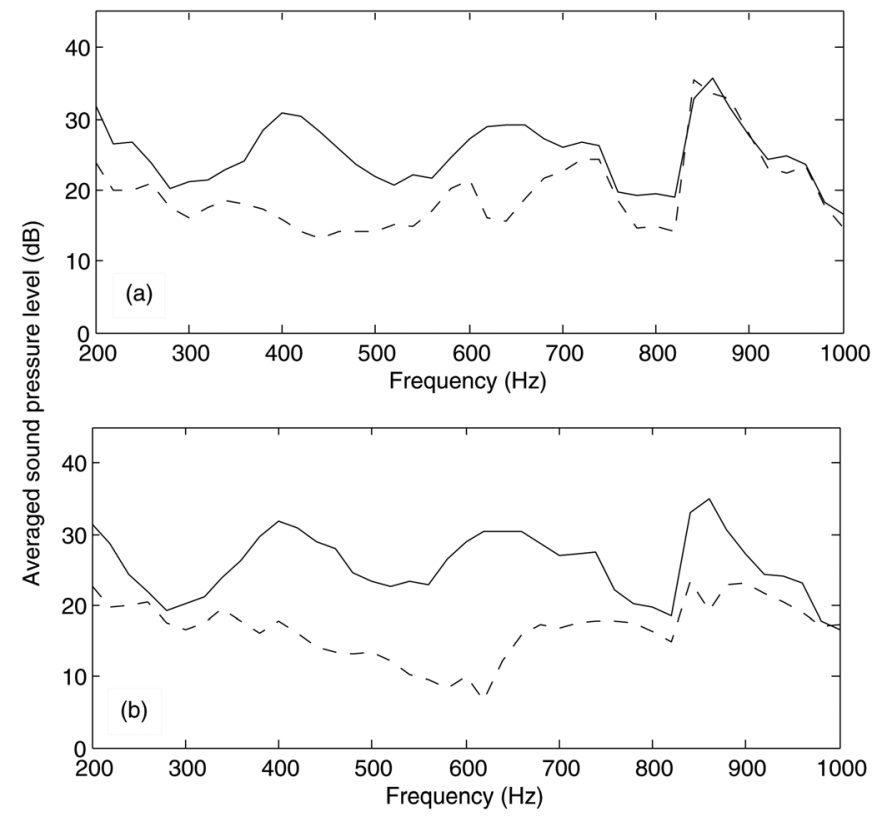

Active noise control off --- Active noise control on

FIG. 12. Averaged SPLs measured with the ANC system turned on or off. A single or a double channel controller was used.

are almost the same. From 300 to $820 \mathrm{~Hz}$, the EA due to the double channel ANC system is greater than that of the single channel ANC system. From 820 to $980 \mathrm{~Hz}$, no effective control can be obtained for the single channel ANC system, while a positive EA can still be achieved for the double channel system. The above results show that the frequency range of effective control as well as the $\mathrm{EA}_{\mathrm{t}}$ can be increased by using a multiple channel system compared with a single channel system, as also concluded in the numerical simulations. There are small differences between the experimental and numerical results. The reason might be that the walls of the room and the frames of the window are assumed to be rigid in the numerical simulations while in the experiments, only a $10 \mathrm{~dB}$ difference was guaranteed, as shown in Fig. 9. Moreover, the secondary sources are assumed to be point sources while in the experiments, the secondary sources are loudspeakers with finite dimensions.

\section{CONCLUSIONS}

An analytical model for calculating the sound field inside a ventilation window and the accompanying room was proposed. In the model, the external free space with traffic noise was approximated by a semi-infinitely long duct with plane wave incidence and the opening sash elements of the window system were regarded as cavities, the mode expansion technique and the coupled cavities theory were employed to solve the sound field inside the natural ventilation window and the room. It was shown that the results obtained with the proposed method agree well with those obtained by the FEM.

The feasibility of applying ANC techniques on such a window system was studied numerically and experimentally. The results of numerical simulations show that the optimal 
arrangement of the secondary sources is placing them at the bottom central position of the staggered window system. For the single channel ANC system investigated in this paper, the frequency range of effective control is up to $390 \mathrm{~Hz}$ in the numerical simulations. For a multi channel ANC system, a wider frequency range of effective control can be achieved, as shown by the numerical simulation results, which is up to 420 $\mathrm{Hz}$ for a double channel ANC system. Moreover, when the primary sound field is well controlled, the extra attenuation at the observation points is almost equivalent to the noise reduction at the error sensors, which is chosen to be $20 \mathrm{~dB}$ in the numerical simulations and $10 \mathrm{~dB}$ in the experiments.

In this paper, some idealized conditions have been assumed, and future work could be to develop a model for more practical situations, such as that in street canyons or squares, ${ }^{26-28}$ where the effects of multiple reflections should be taken into account, as well as the effects of air flow.

\section{ACKNOWLEDGMENTS}

The authors are grateful to the anonymous reviewers for their constructive comments on improving the original manuscript. The project is partially supported by NSFC (11004101) and the Fundamental Research Funds for the Central Universities. The first author was supported by the Chinese Scholarship Council for a visiting research at the University of Sheffield. The third author is indebted to the NSFC (50928801) for the support.

\section{APPENDIX}

For the eigenfunctions, there are

$$
\begin{aligned}
& \int_{S_{l}} \varphi_{l}^{m_{l} n_{l}}\left(y_{l}, z_{l}\right) d S=0, \text { except for } m_{l}=n_{l}=0, \\
& \int_{S_{l}} \varphi_{l}^{m_{l} n_{l}}\left(y_{l}, z_{l}\right) \times \varphi_{l}^{m_{l}^{\prime} n_{l}^{\prime}}\left(y_{l}, z_{l}\right) d S=\Lambda_{m_{l}^{\prime} n_{l}^{\prime}} \delta_{m_{l} n_{l}, m_{l}^{\prime} n_{l}^{\prime}},
\end{aligned}
$$

where the integral is taken over the cavity cross section. $\delta_{m_{l} n_{l}, m_{l}^{\prime} n_{l}^{\prime}}$ is Kronecker $\delta$.

$$
\Lambda_{m_{l}^{\prime} n_{l}^{\prime}}=\int_{S_{l}}\left[\varphi_{l}^{m_{l}^{\prime} n_{l}^{\prime}}\left(y_{l}, z_{l}\right)\right]^{2} d S
$$

is the norm of eigenfunction for mode $\left(m_{l}^{\prime}, n_{l}^{\prime}\right)$.

For Eq. (4), multiplying it by $\varphi_{1}^{m_{1}^{\prime} n_{1}^{\prime}}\left(y_{1}, z_{1}\right)$ and integrating it over the cross section of cavity $\mathrm{V}_{1}\left(S_{1}\right)$ will give

$$
\begin{aligned}
& \sum_{m_{1}=0}^{\infty} \sum_{n_{1}=0}^{\infty} P_{i_{1}, x_{1}=0}^{m_{1} n_{1}}\left\langle\varphi_{1}^{m_{1} n_{1}}\left(y_{1}, z_{1}\right) \varphi_{1}^{m_{1}^{\prime} n_{1}^{\prime}}\left(y_{1}, z_{1}\right)\right\rangle_{S_{1}} \\
& \quad=P_{0}\left\langle\varphi_{1}^{m_{1}^{\prime} n_{1}^{\prime}}\left(y_{1}, z_{1}\right)\right\rangle_{S_{1}}
\end{aligned}
$$

where \langle\rangle$_{s}$ denotes integration of the expression inside the parenthesis over the area indicated at the subscript. From Eq. (A1), it can be derived that the integration on the r.h.s. of Eq. (A4) equals 0 except $m_{1}^{\prime}=n_{1}^{\prime}=0$, which leads to that for the 1.h.s. of Eq. (A4)

$$
P_{i_{1}, x_{1}=0}^{m_{1} n_{1}}=0, \text { except for } m_{1}=n_{1}=0 .
$$

This means that only $(0,0)$ mode exists in the incident wave in cavity $\mathrm{V}_{1}$, corresponding to the assumed normal plane wave incidence condition.

For Eq. (5), multiplying it by $\varphi_{5}^{m_{5}^{\prime} n_{5}^{\prime}}\left(y_{5}, z_{5}\right)$ and integrating it over the cross section of cavity $\mathrm{V}_{5}\left(S_{5}\right)$ will give

$$
\begin{aligned}
& \sum_{m_{5}=0}^{\infty} \sum_{n_{5}=0}^{\infty} K_{x_{5}}^{m_{5} n_{5}}\left(P_{i_{5}, x_{5}=L_{x_{5}}}^{m_{5} n_{5}}-P_{r_{5}, x_{5}=L_{x_{5}}}^{m_{5} n_{5}}\right) \\
& \quad \times\left\langle\varphi_{5}^{m_{5} n_{5}}\left(y_{5}, z_{5}\right) \varphi_{5}^{m_{5}^{\prime} n_{5}^{\prime}}\left(y_{5}, z_{5}\right)\right\rangle_{S_{5}}=0
\end{aligned}
$$

and it characterizes the rigid boundary condition at plane $\mathrm{F}$.

For Eq. (7a), multiplying it by $\varphi_{1}^{m_{1}^{\prime} n_{1}^{\prime}}\left(y_{1}, z_{1}\right)$ and integrating it over the interface area of cavity $\mathrm{V}_{1}$ and cavity $\mathrm{V}_{2}\left(S_{12}\right)$ :

$$
\begin{aligned}
& \sum_{m_{1}=0}^{\infty} \sum_{n_{1}=0}^{\infty}\left(P_{i_{1}, x_{1}=L_{x_{1}}}^{m_{1} n_{1}}+P_{r_{1}, x_{1}=L_{x_{1}}}^{m_{1} n_{1}}\right)\left\langle\varphi_{1}^{m_{1} n_{1}}\left(y_{1}, z_{1}\right) \varphi_{1}^{m_{1}^{\prime} n_{1}^{\prime}}\left(y_{1}, z_{1}\right)\right\rangle_{S_{12}} \\
& =\sum_{m_{2}=0}^{\infty} \sum_{n_{2}=0}^{\infty}\left(P_{i_{2}, x_{2}=0}^{m_{2} n_{2}}+P_{r_{2}, x_{2}=0}^{m_{2} n_{2}}\right)\left\langle\varphi_{2}^{m_{2} n_{2}}\left(y_{2}, z_{2}\right) \varphi_{1}^{m_{1}^{\prime} n_{1}^{\prime}}\left(y_{1}, z_{1}\right)\right\rangle_{S_{2}} .
\end{aligned}
$$

Equation (A7) represents the continuity of the acoustic pressure at the interface of cavity $\mathrm{V}_{1}$ and cavity $\mathrm{V}_{2}$.

The continuity of normal velocity at the interface in plane B requires Eq. (7b). Furthermore, the normal velocity on the wall of cavity $V_{1}$ in plane $B$ is

$$
v_{w, 1}^{B}=v_{1}^{B}=0
$$

The nonhomogeneous boundary conditions between cavity $\mathrm{V}_{1}$ and $\mathrm{V}_{2}$ can be expressed by the continuity conditions, which include the sound pressure continuity, represented by Eq. (7a), and the volume velocity continuity, represented by Eqs. (7b) and (A8). Multiplying Eqs. (7b) and (A8) by $\varphi_{1}^{m_{1}^{\prime} n_{1}^{\prime}}\left(y_{1}, z_{1}\right)$ and, integrating it over $S_{12}$ and $\left(S_{1}-S_{12}\right)$, respectively:

$$
\begin{aligned}
& \int_{S_{12}}\left(v_{1}^{i}+v_{1}^{r}\right) \varphi_{1}^{m_{1}^{\prime} n_{1}^{\prime}}\left(y_{1}, z_{1}\right) d S \\
& =\int_{S_{12}}\left(v_{2}^{i}+v_{2}^{r}\right) \varphi_{1}^{m_{1}^{\prime} n_{1}^{\prime}}\left(y_{1}, z_{1}\right) d S, \\
& \int_{S_{1}-S_{12}}\left(v_{1}^{i}+v_{1}^{r}\right) \varphi_{1}^{m_{1}^{\prime} n_{1}^{\prime}}\left(y_{1}, z_{1}\right) d S \\
& =\int_{S_{1}-S_{12}} v_{w, 1}^{B} \varphi_{1}^{m_{1}^{\prime} n_{1}^{\prime}}\left(y_{1}, z_{1}\right) d S,
\end{aligned}
$$

Adding Eqs. (A9) and (A10) together gives 


$$
\begin{aligned}
& \int_{S_{1}}\left(v_{1}^{i}+v_{1}^{r}\right) \varphi_{1}^{m_{1}^{\prime} n_{1}^{\prime}}\left(y_{1}, z_{1}\right) d S \\
&=\int_{S_{12}}\left(v_{2}^{i}+v_{2}^{r}\right) \varphi_{1}^{m_{1}^{\prime} n_{1}^{\prime}}\left(y_{1}, z_{1}\right) d S .
\end{aligned}
$$

Expressing $v_{1}^{i}, v_{1}^{r}, v_{2}^{i}$ and $v_{2}^{r}$ in the form given by Eq. (6) and inserting it back into Eq. (A11), and then one finally has

$$
\begin{aligned}
\sum_{m_{1}=0}^{\infty} \sum_{n_{1}=0}^{\infty} K_{x_{1}}^{m_{1} n_{1}}\left(P_{i_{1}, x_{1}=L_{x_{1}}}^{m_{1} n_{1}}-P_{r_{1}, x_{1}=L_{x_{1}}}^{m_{1} n_{1}}\right) \\
\times\left\langle\varphi_{1}^{m_{1} n_{1}}\left(y_{1}, z_{1}\right) \varphi_{1}^{m_{1}^{\prime} n_{1}^{\prime}}\left(y_{1}, z_{1}\right)\right\rangle_{S_{1}} \\
=\sum_{m_{2}=0}^{\infty} \sum_{n_{2}=0}^{\infty} K_{x_{2}}^{m_{2} n_{2}}\left(P_{i_{2}, x_{2}=0}^{m_{2} n_{2}}-P_{r_{2}, x_{2}=0}^{m_{2} n_{2}}\right) \\
\times\left\langle\varphi_{2}^{m_{2} n_{2}}\left(y_{2}, z_{2}\right) \varphi_{1}^{m_{1}^{\prime} n_{1}^{\prime}}\left(y_{1}, z_{1}\right)\right\rangle_{S_{2}} .
\end{aligned}
$$

Equation (A12) represents the continuity of volume velocity in plane B. Equations for other continuity conditions corresponding to Eqs. (8), (9), and (10) can be derived in a similar way and the results can be given directly.

For Eq. (8a), the continuity of acoustic pressure in plane $\mathrm{C}$ requires

$$
\begin{aligned}
& \sum_{m_{2}=0}^{\infty} \sum_{n_{2}=0}^{\infty}\left(P_{i_{2}, x_{2}=L_{x_{2}}}^{m_{2} n_{2}}+P_{r_{2}, x_{2}=L_{x_{2}}}^{m_{2} n_{2}}\right) \\
& \times\left\langle\varphi_{2}^{m_{2} n_{2}}\left(y_{2}, z_{2}\right) \varphi_{3}^{m_{3}^{\prime} n_{3}^{\prime}}\left(y_{3}, z_{3}\right)\right\rangle_{S_{2}} \\
&=\sum_{m_{3}=0}^{\infty} \sum_{n_{3}=0}^{\infty}\left(P_{i_{3}, x_{3}=0}^{m_{3} n_{3}}+P_{r_{3}, x_{3}=0}^{m_{3} n_{3}}\right) \\
& \times\left\langle\varphi_{3}^{m_{3} n_{3}}\left(y_{3}, z_{3}\right) \varphi_{3}^{m_{3}^{\prime} n_{3}^{\prime}}\left(y_{3}, z_{3}\right)\right\rangle_{S_{23}} .
\end{aligned}
$$

For Eq. (8b), the continuity of volume velocity in plane C requires

$$
\begin{aligned}
\sum_{m_{2}=0}^{\infty} \sum_{n_{2}=0}^{\infty} K_{x_{2}}^{m_{2} n_{2}}\left(P_{i_{2}, x_{2}=L_{x_{2}}}^{m_{2} n_{2}}-P_{r_{2}, x_{2}=L_{x_{2}}}^{m_{2} n_{2}}\right) \\
\times\left\langle\varphi_{2}^{m_{2} n_{2}}\left(y_{2}, z_{2}\right) \varphi_{3}^{m_{3}^{\prime} n_{3}^{\prime}}\left(y_{3}, z_{3}\right)\right\rangle_{S_{2}} \\
=\sum_{m_{3}=0}^{\infty} \sum_{n_{3}=0}^{\infty} K_{x_{3}}^{m_{3} n_{3}}\left(P_{i_{3}, x_{3}=0}^{m_{3} n_{3}}-P_{r_{3}, x_{3}=0}^{m_{3} n_{3}}\right) \\
\times\left\langle\varphi_{3}^{m_{3} n_{3}}\left(y_{3}, z_{3}\right) \varphi_{3}^{m_{3}^{\prime} n_{3}^{\prime}}\left(y_{3}, z_{3}\right)\right\rangle_{S_{3}} .
\end{aligned}
$$

For Eq. (9a), the continuity of acoustic pressure in plane D requires

$$
\begin{aligned}
\sum_{m_{3}=0}^{\infty} \sum_{n_{3}=0}^{\infty}\left(P_{i_{3}, x_{3}=L_{x_{3}}}^{m_{3} n_{3}}\right. & \left.+P_{r_{3}, x_{3}=L_{x_{3}}}^{m_{3} n_{3}}\right)\left\langle\varphi_{3}^{m_{3} n_{3}}\left(y_{3}, z_{3}\right) \varphi_{3}^{m_{3}^{\prime} n_{3}^{\prime}}\left(y_{3}, z_{3}\right)\right\rangle_{S_{34}} \\
=\sum_{m_{4}=0}^{\infty} \sum_{n_{4}=0}^{\infty}\left(P_{i_{4}, x_{4}=0}^{m_{4} n_{4}}+P_{r_{4}, x_{4}=0}^{m_{4} n_{4}}\right) & \\
& \times\left\langle\varphi_{4}^{m_{4} n_{4}}\left(y_{4}, z_{4}\right) \varphi_{3}^{m_{3}^{\prime} n_{3}^{\prime}}\left(y_{3}, z_{3}\right)\right\rangle_{S_{4}} . \quad \text { (A15) }
\end{aligned}
$$

For Eq. (9b), the continuity of volume velocity in plane D requires

$$
\begin{aligned}
& \sum_{m_{3}=0}^{\infty} \sum_{n_{3}=0}^{\infty} K_{x_{3}}^{m_{3} n_{3}}\left(P_{i_{3}, x_{3}=L_{x_{3}}}^{m_{3} n_{3}}-P_{r_{3}, x_{3}=L_{x_{3}}}^{m_{3} n_{3}}\right) \\
& \times\left\langle\varphi_{3}^{m_{3} n_{3}}\left(y_{3}, z_{3}\right) \varphi_{3}^{m_{3}^{\prime} n_{3}^{\prime}}\left(y_{3}, z_{3}\right)\right\rangle_{S_{3}} \\
&=\sum_{m_{4}=0}^{\infty} \sum_{n_{4}=0}^{\infty} K_{x_{4}}^{m_{4} n_{4}}\left(P_{i_{4}, x_{4}=0}^{m_{4} n_{4}}-P_{r_{4}, x_{4}=0}^{m_{4} n_{4}}\right) \\
& \times\left\langle\varphi_{4}^{m_{4} n_{4}}\left(y_{4}, z_{4}\right) \varphi_{3}^{m_{3}^{\prime} n_{3}^{\prime}}\left(y_{3}, z_{3}\right)\right\rangle_{S_{4}} .
\end{aligned}
$$

For Eq. (10a), the continuity of acoustic pressure in plane E requires

$$
\begin{gathered}
\sum_{m_{4}=0}^{\infty} \sum_{n_{4}=0}^{\infty}\left(P_{i_{4}, x_{4}=L_{x_{4}}}^{m_{4} n_{4}}+P_{r_{4}, x_{4}=L_{x_{4}}}^{m_{4} n_{4}}\right)\left\langle\varphi_{4}^{m_{4} n_{4}}\left(y_{4}, z_{4}\right) \varphi_{5}^{m_{5}^{\prime} n_{5}^{\prime}}\left(y_{5}, z_{5}\right)\right\rangle_{S_{4}} \\
=\sum_{m_{5}=0}^{\infty} \sum_{n_{5}=0}^{\infty}\left(P_{i_{5}, x_{5}=0}^{m_{5} n_{5}}+P_{r_{5}, x_{5}=0}^{m_{5} n_{5}}\right) \\
\quad \times\left\langle\varphi_{5}^{m_{5} n_{5}}\left(y_{5}, z_{5}\right) \varphi_{5}^{m_{5}^{\prime} n_{5}^{\prime}}\left(y_{5}, z_{5}\right)\right\rangle_{S_{45}}
\end{gathered}
$$

For Eq. (10b), the continuity of volume velocity in plane E requires

$$
\begin{aligned}
\sum_{m_{4}=0}^{\infty} \sum_{n_{4}=0}^{\infty} K_{x_{4}}^{m_{4} n_{4}}\left(P_{i_{4}, x_{4}=L_{x_{4}}}^{m_{4} n_{4}}-P_{r_{4}, x_{4}=L_{x_{4}}}^{m_{4} n_{4}}\right) \\
\times\left\langle\varphi_{4}^{m_{4} n_{4}}\left(y_{4}, z_{4}\right) \varphi_{5}^{m_{5}^{\prime} n_{5}^{\prime}}\left(y_{5}, z_{5}\right)\right\rangle_{S_{4}} \\
=\sum_{m_{5}=0}^{\infty} \sum_{n_{5}=0}^{\infty} K_{x_{5}}^{m_{5} n_{5}}\left(P_{i_{5}, x_{5}=0}^{m_{5} n_{5}}-P_{r_{5}, x_{5}=0}^{m_{5} n_{5}}\right) \\
\times\left\langle\varphi_{5}^{m_{5} n_{5}}\left(y_{5}, z_{5}\right) \varphi_{5}^{m_{5}^{\prime} n_{5}^{\prime}}\left(y_{5}, z_{5}\right)\right\rangle_{S_{5}} .
\end{aligned}
$$

Equations (A4), (A6), and (A7) and Eqs. (A12) to (A18) constitute a set of linear equations which fully describes the sound field of the window-room system. Noting that there are four groups of unknown modal amplitudes, namely, $P_{i_{l}, x_{l}=0}^{m_{l} n_{l}}, P_{i_{l}, x_{l}=L_{x_{l}}}^{m_{1} n_{l}}, P_{r_{l}, x_{l}=0}^{m_{l} n_{l}}$ and $P_{r_{l}, x_{l}=L_{x_{l}}}^{m_{l} n_{l}}$, meanwhile they have the relationships of

$$
\begin{aligned}
& P_{i_{l}, x_{l}=L_{x_{l}}}^{m_{l} n_{l}}=P_{i_{l}, x_{l}=0}^{m_{l} n_{l}} \exp \left(-j k_{x_{l}}^{m_{l} n_{l}} L_{x_{l}}\right), \\
& P_{r_{l}, x_{l}=L_{x_{l}}}^{m_{l} n_{l}}=P_{r_{l}, x_{l}=0}^{m_{l} n_{l}} \exp \left(j k_{x_{l}}^{m_{l} n_{l}} L_{x_{l}}\right),
\end{aligned}
$$

with which the unknowns can be reduced to $P_{i_{l}, x_{l}=0}^{m_{l} n_{l}}$ and $P_{r_{l}, x_{l}=0}^{m_{l} n_{l}}$ in the above equations. Writing the set of linear equations in matrix form and one can get Eq. (12) as

$$
\mathbf{A P}=\mathbf{C},
$$


where

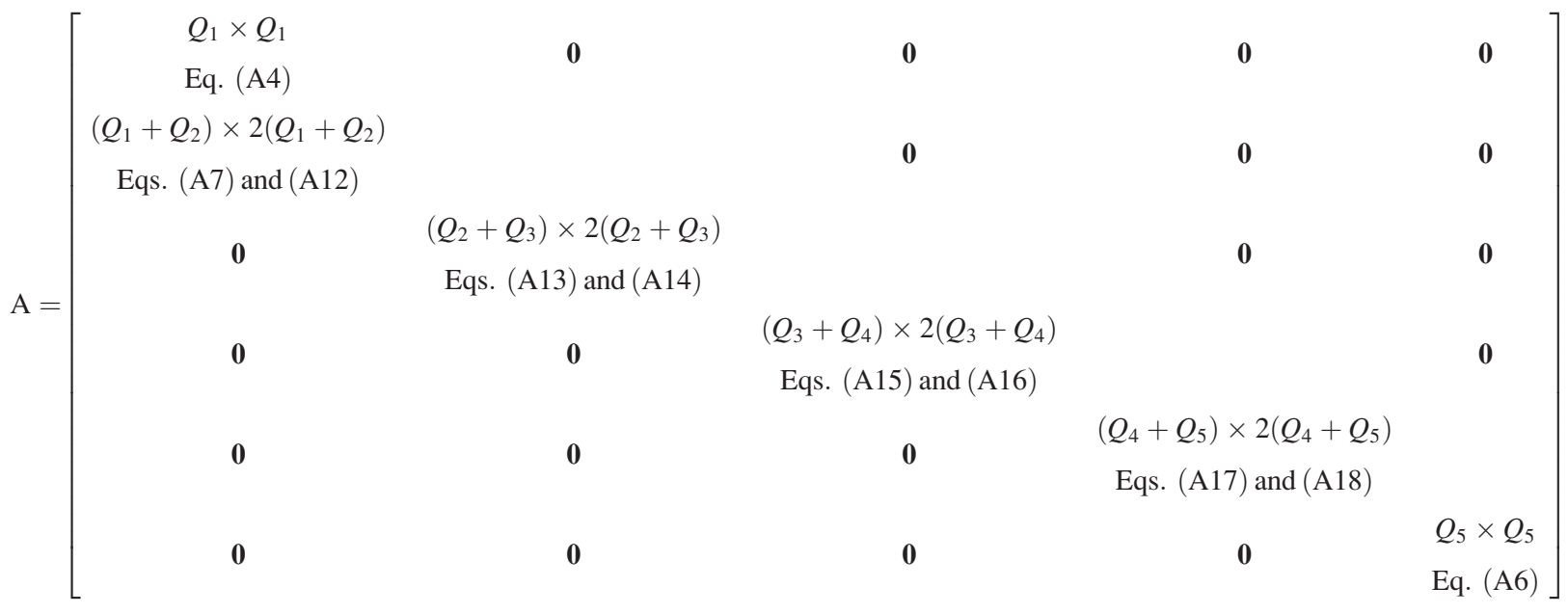

is a $2 Q_{0} \times 2 Q_{0}$ matrix of coefficients.

${ }^{1}$ E. Öhrström, L. Barregård, E. Andersson, A. Skånberg, H. Svensson, and P. Ängerheim, "Annoyance due to single and combined sound exposure from railway and road traffic," J. Acoust. Soc. Am. 122, 2642-2652 (2007).

${ }^{2}$ R. D. Ford and G. Kerry, "The sound insulation of partially open double glazing,” Appl. Acoust. 6, 57-72 (1973).

${ }^{3}$ A. B. Lawrence and M. A. Burgess, "Traffic noise and open window (A)," J. Acoust. Soc. Am. 72, S91 (1982).

${ }^{4} \mathrm{~F}$. Cotana, "Experimental data and performances of new high sound insulation ventilating windows," Proceedings of Inter-Noise 1999, FL, pp. 995-998.

${ }^{5}$ C. D. Field and F. R. Fricke, "Theory and applications of quarter-wave resonators: a prelude to their use for attenuating noise entering buildings through openings," Appl. Acoust. 53, 117-132 (1998).

${ }^{6}$ S. K. Tang, D. Suen, and S. K. Chan, "Parametric study of the sound insulation performance of a ventilation window (A)," J. Acoust. Soc. Am. 127, 1776 (2010).

${ }^{7}$ Y. Nishimura, S. Nishimura, T. Nishimura, and T. Yano, "Sound propagation in soundproofing casement windows," Appl. Acoust. 70, 1160-1167 (2009).

${ }^{8}$ J. Kang and M. W. Brocklesby, "Feasibility of applying micro-perforated absorbers in acoustic window systems," Appl. Acoust. 66, 669-689 (2005).

${ }^{9}$ J. Kang and Z. Li, "Numerical simulation of an acoustic window system using finite element method," Acust. Acta Acust. 93, 152-163 (2007).

${ }^{10}$ J. Guo and J. Pan, "Increasing the insertion loss of noise barriers using an active-control system," J. Acoust. Soc. Am. 104, 3408-3416 (1998).

${ }^{11}$ J. Zhang, W. Jiang, and N. Li, "Theoretical and experimental investigations on coherence of traffic noise transmission through an open window into a rectangular room in high-rise buildings," J. Acoust. Soc. Am. 112, 1482-1495 (2002).

${ }^{12}$ A. Jakob and M. Möser, "Active control of double-glazed windows. Part I: Feedforward control,” Appl. Acoust. 64, 163-182 (2003).

${ }^{13}$ A. Jakob and M. Möser, "Active control of double-glazed windows. Part II: Feedback control," Appl. Acoust. 64, 183-196 (2003).
${ }^{14}$ S. Ise, "The boundary surface control principle and its applications," IEICE Trans. Fundamentals E88-A, 1656-1664 (2005).

${ }^{15}$ R. Mittra and S. W. Lee, Analytical Techniques in the Theory of Guided Waves (Macmillan, New York, 1971), Chaps. 2 and 4.

${ }^{16} \mathrm{M}$. Åbom, "Derivation of four-pole parameters including higher order mode effects for expansion chamber mufflers with extended inlet and outlet," J. Sound Vib. 137, 403-418 (1990).

${ }^{17}$ R. D. Ford, "Predicting the sound reduction index of laminated glass," Appl. Acoust. 43, 49-55 (1994).

${ }^{18}$ W. Kropp and J. Bérillon, "A theoretical model to investigate the acoustic performance of building facades in the low and middle frequency range," Acust. Acta Acust. 84, 681-688 (1998).

${ }^{19}$ A. I. El-Sharkawy and A. H. Nayfeh, "Effect of an expansion chamber on the propagation of sound in circular ducts," J. Acoust. Soc. Am. 63, 667-674 (1978).

${ }^{20}$ R. Glav, P.-L. Regaud, and M. Åbom, "Study of a folded resonator including the effects of higher order modes," J. Sound Vib. 273, 777-792 (2004).

${ }^{21}$ P. M. Morse and K. U. Ingard, Theoretical Acoustics (McGraw-Hill, New York, 1968), Chaps. 9 and 10.

${ }^{22}$ P. Jean, "Sound transmission through opened windows," Appl. Acoust. 70, 41-49 (2009).

${ }^{23}$ P. A. Nelson and S. J. Elliott, Active Control of Sound (Academic, London, 1992), Chap. 8.

${ }^{24}$ COMSOL user's guide (Acoustics Module), COMSOL AB, Sweden, 2008.

${ }^{25} \mathrm{H}$. Zou, X. Qiu, J. Lu, and F. Niu, “A preliminary experimental study on virtual sound barrier system,” J. Sound Vib. 307, 379-385 (2007).

${ }^{26} \mathrm{~J}$. Kang, "Sound propagation in interconnected urban streets: a parametric study," Environ. Plann. B 28, 281-294 (2001).

${ }^{27} \mathrm{~J}$. Kang, "Numerical modeling of the sound fields in urban squares," J. Acoust. Soc. Am. 117, 3695-3706 (2005).

${ }^{28}$ J. Kang, "Numerical modelling of the sound fields in urban streets with diffusely reflecting boundaries," J. Sound Vib. 258, 793-813 (2002). 